\title{
A New Directional Canopy Emissivity Model Based on Spectral Invariants
}

\author{
Biao $\mathrm{Cao}^{\circledR}$, Mingzhu Guo, Wenjie Fan, Xiru Xu, Jingjing Peng ${ }^{\circledR}$, Huazhong Ren ${ }^{\circledR}$, \\ Yongming Du, Hua $\mathrm{Li}^{\circledR}$, Zunjian Bian $\left.{ }^{(}\right)$, Tian $\mathrm{Hu}^{\circledR}$, Qing Xiao ${ }^{(}$, and Qinhuo Liu ${ }^{(}$
}

\begin{abstract}
A new directional canopy emissivity model (CE-P) based on spectral invariants is proposed in this paper. First, we prove the existence of the spectral invariant properties in the thermal infrared (TIR) band using a Monte Carlo model. Based on it, the equation of the new model is derived from the perspective of absorption. In this expression, single-scattering and multiscattering effects are separated analytically in the TIR band. We find that the overall contribution of multiple scatterings is less than 0.005 when the component emissivities are over 0.90 , and the overall contribution decreases with increasing leaf or soil emissivity. Furthermore, the new model can avoid the logical difficulty encountered when using the traditional cavity effect factor to simulate the emissivity of a sparse vegetation canopy. The results of 4SAIL and Discrete Anisotropic Radiative Transfer (DART) are selected to do cross validation. The CE-P can achieve a high accuracy compared with 4SAIL and DART, with an absolute bias less than 0.002 when the leaf (soil) emissivity is equal to $0.98(0.94)$. Four widely used analytical models are selected for comparison. The resulting accuracies of these models are ordered from CE-P to REN15, FR97, FR02, and VALOR96 with the most serious error up to $0.002,0.002$, $0.007,0.013$, and 0.014 , respectively. Three main conclusions are obtained through the sensitivity analysis: the multiscattering between vegetation and the background can be ignored when the leaf (soil) emissivity is no less than $0.94(0.90)$, the second and higher order scattering within the vegetation can also be ignored when the leaf (soil) emissivity is no less than $0.94(0.90)$, and the single-scattering effect within the canopy should be considered which can be calculated using three view factors.
\end{abstract}

Index Terms-Canopy emissivity, land surface emissivity, land surface temperature (LST), recollision probability, spectral invariants.

\section{NOMENCLATURE}

$\alpha \quad$ Cavity effect factor.

$M \quad$ Number of absorbed photons.

Manuscript received December 1, 2017; revised February 11, 2018 and June 2, 2018; accepted June 4, 2018. This work was supported in part by the National Natural Science of Foundation of China under Grant 41571329 , Grant 41501366, Grant 41571357, Grant 41571359, and Grant 41671366, and in part by the Director Foundation of the Institute of Remote Sensing and Digital Earth, Chinese Academy of Sciences, under Grant Y5SJ1300CX. (Corresponding authors: Wenjie Fan; Yongming Du.)

B. Cao, Y. Du, H. Li, Z. Bian, T. Hu, Q. Xiao, and Q. Liu are with the State Key Laboratory of Remote Sensing Science, Institute of Remote Sensing and Digital Earth, Chinese Academy of Sciences, Beijing 100101, China, and also with the Joint Center for Global Change Studies, Beijing 100875, China (e-mail: ymdushandong@foxmail.com).

M. Guo, W. Fan, X. Xu, and H. Ren are with the Institute of Remote Sensing and Geographic Information System, Peking University, Beijing 100871, China (e-mail: fanwj@pku.edu.cn).

J. Peng is with the University of Maryland, College Park, MD 20740 USA. Digital Object Identifier 10.1109/TGRS.2018.2845678
$N \quad$ Number of incident photons.

$\theta \quad$ View zenith angle.

$p \quad$ Recollision probability.

$\rho_{\mathrm{dh}} \quad$ Directional-hemispherical reflectance.

$e \quad$ Directional canopy emissivity.

$e_{u} \quad$ Upward escape probability.

$e_{d} \quad$ Downward escape probability.

$a_{u} \quad$ The probability of a photon moving upward after being scattered by a leaf.

$a_{d} \quad$ The probability of a photon moving downward after being scattered by a leaf.

$a \quad$ Directional canopy absorption.

av Vegetation directional absorptance.

as Background directional absorptance.

$b_{0} \quad$ Canopy gap fraction.

$i_{0} \quad$ Canopy interception.

$i_{0}^{\prime} \quad$ Hemispherical average intercept probability.

$u_{l} \quad$ Leaf area density.

$H \quad$ Canopy height.

$h \quad$ The height of a layer.

$d h \quad$ The thickness of a layer.

$G(\theta)$ The fraction of leaves projected in the direction $\theta$.

$\Omega \quad$ Clumping index.

$w \quad$ Single-scattering albedo.

$\varepsilon_{v} \quad$ Leaf emissivity.

$\varepsilon_{s} \quad$ Background emissivity.

$\varepsilon_{\text {lim }} \quad$ Limited canopy emissivity with very large LAI.

$r_{c 1}^{*} \quad$ Forward diffuse reflectance of the canopy.

$r_{c 2}^{*} \quad$ Backward diffuse reflectance of the canopy.

$d \varepsilon \quad$ Scattering effect in VALOR96 model.

$\varepsilon 1 \quad$ Leaf emission.

$\varepsilon 2$ Leaf reflected radiance coming from the leaf emission.

$F_{l l} \quad$ View factor of $\varepsilon 2$.

\&3 Soil reflected radiance coming from the leaf emission.

$F_{l s} \quad$ View factor of $\varepsilon 3$.

$\varepsilon 4 \quad$ Soil emission.

\&5 Leaf reflected radiance coming from the soil emission.

$F_{s l} \quad$ View factor of $\varepsilon 5$.

\section{INTRODUCTION}

$\mathbf{L}$ AND surface temperature (LST) is one of the most important parameters in the physical processes of surface energy and water balance at both local and global scales [1]. 
It is the direct driving force of turbulent heat fluxes at the surface and atmosphere interface and is widely used in the fields of evapotranspiration estimation and hydrological cycle [2]. Remote sensing products offer the only possibility for measuring LSTs with completely spatially averaged values [3].

The angular effect of LSTs has been widely concerned in the area of thermal infrared (TIR) remote sensing. Many models have been proposed to simulate the directional brightness temperature (DBT) distribution and land surface emissivity distribution over different underlying surfaces since the 1960s. The underlying surfaces, such as vegetation [4]-[8], bare soil [9], [10], urban [11], [12], snow, and water [13], have been widely discussed. Furthermore, the DBT distribution of mixed pixels has also been discussed in recent years [14], [15].

Vegetation is the one of the most important ecosystems of the land surface. Many models have been proposed to simulate the directional emissivities of vegetation canopies [16], and these models can be divided into three categories: geometrical models, radiative transfer models (RTMs), and ray tracing models. For homogeneous canopies, RTMs (such as S\&WVM [17], FR97 [18], FR02 [19], and 4SAIL [20]) are used more widely. In 2015, Ren et al. [21] proposed a new homogeneous canopy directional emissivity model (REN15) and further compared it with $\mathrm{S} \& \mathrm{~W}^{\mathrm{VM}}$, FR97, FR02, and 4SAIL. REN15 can obtain the most accurate results while the 4SAIL was treated as reference, especially for large view zenith angles (VZAs).

It is widely accepted that FR02 is always underestimated because it ignores the multiple-scattering effects within vegetation layers. The 4SAIL can produce the most accurate emissivities which can be used as reference to validate other models, but the form of differential equations leads to the parameter inversion largely depending on the lookup table. Therefore, REN15 and FR97 are widely used for parameter inversion. The equations of these two models are similar, but the unique difference between them is the values of the lookup table of $\alpha$, which is an indicator of the multiple scatterings inside the canopy. The $\alpha$ of FR97 is calculated based on the Prevot model [22], and that of REN15 is obtained from 4SAIL. A ray tracing model [such as the Discrete Anisotropic Radiative Transfer (DART)] [23], [24] is always used for validation because of its high accuracy. However, a ray tracing model is not suitable for parameter inversion because of its high time cost.

François et al. [18] found that the canopy directional emissivity at a specified VZA always reaches a limit emissivity that depends only on the vegetation emissivity and VZA rather than on the background emissivity. The canopy limit emissivity is larger than the leaf emissivity due to multiple scatterings. François et al. [18] developed $\alpha$ parameter to represent the cavity effect based on the canopy limit emissivity and leaf emissivity. However, if the cavity effect factor is used to calculate the directional emissivity of the canopy with small leaf area index (LAI), a logical difficulty arises because the factor can be calculated only when the LAI is very large (under extreme conditions). In fact, $\alpha$ should be not only angle-dependent but also LAI-dependent, which means that the cavity effect should change with LAI and canopy architecture (i.e., LAI 3-D distribution).

For the bidirectional reflectance distribution function simulation in the visible and near infrared (VNIR) bands, multiple scatterings are also a problem that needs to be solved. Spectral invariants (such as the recollision probability value $p$ ) provide an opportunity to give an analytical solution of the multiplescattering effect in the VNIR band [25]. However, no evidence can currently support the definition of spectral invariants in the TIR band, so few studies extend the concept to the TIR band to evaluate the multiscattering effect in the TIR band.

In this paper, we tried to extend the spectral invariants in the TIR band and to propose a new analytical method to calculate the cavity effect within a homogeneous canopy based on spectral invariants. Based on this method, a new physical analytical model will be developed to simulate the directional emissivity of a homogeneous canopy. The contributions of leaves and soil can be directly separated in this model without the use of the empirical factor $\alpha$. The methodology is introduced in Section II. Section III presents the sensitivity analysis results. The cross validation and comparison are given in Sections IV and V. Section VI presents the discussion and conclusion.

\section{Methodology}

\section{A. Model Basis: Kirchhoff's Law}

Based on Kirchhoff's law, directional canopy emissivity is equal to the difference between one and the directionalhemispherical reflectance and is equal to the absorption in the incident direction. This law is widely applied to measure emissivity in the field and laboratory [26], such as the widely used TIR spectral emissivity libraries of the University of California at Santa Barbara (UCSB, website: http://www.icess.ucsb.edu/modis/EMIS/html/em.html), Santa Barbara, CA, USA.

Assuming there are $N$ photons coming from a given direction $(\theta)$, when the canopy (including the vegetation and background) absorbs $M$ photons, the number of reflected photons should be equal to $N-M$, according to the law of energy conservation. Then, the directional absorption is equal to $M / N$, and the directional-hemispherical reflectance $\left(\rho_{\mathrm{dh}}\right)$ is equal to $(N-M) / N$. Finally, the directional emissivity in this direction $(\theta)$ can be obtained based on Kirchhoff's law, as shown in the following equations:

$$
\begin{aligned}
& e(\theta)=1-\rho_{\mathrm{dh}}=1-\frac{N-M}{N}=\frac{M}{N} \\
& e(\theta)=a(\theta)=\frac{M}{N} .
\end{aligned}
$$

The equation for the directional absorption (containing both vegetation absorption and soil absorption) of a continuous canopy will be introduced in detail in Section II-D, and the equations for the essential parameters, including the recollision probability, interceptions, escape probability, gap fraction, and diffuse reflectance, will be given in Sections II-B and II-C. 


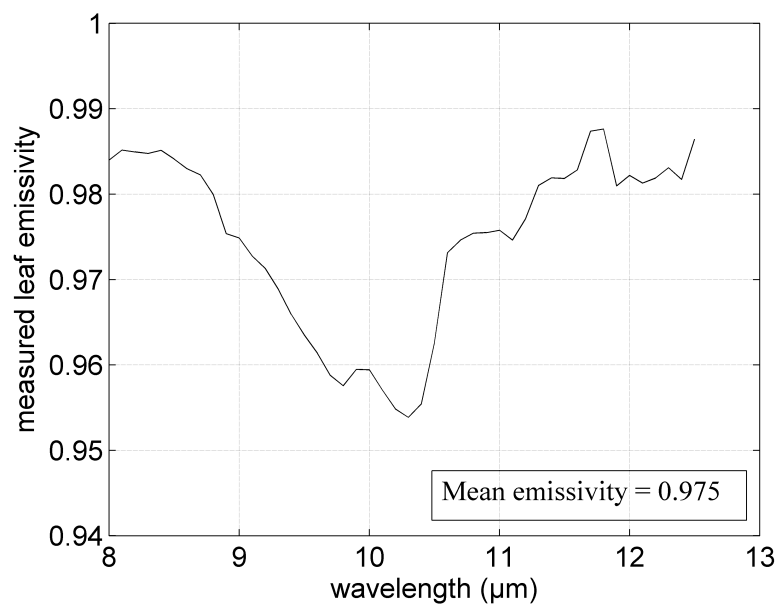

Fig. 1. Measured leaf emissivity in the TIR band $(8-12.5 \mu \mathrm{m})$.

\section{B. Spectral Invariants}

The spectral invariants [27]-[32] [including the recollision probability $(p)$, the canopy interceptions $\left(i_{0}\right)$, and the directional escape probability] are widely used in the VNIR band to simulate the bidirectional reflectance factor. The recollision probability is defined as the probability by which a photon scattered from a leaf or needle in the canopy will interact within the canopy again. The recollision probability is supposed to be independent of wavelength but changes with the LAI and VZA. Stenberg et al. [33] reviewed the recollision probability in modeling in the VNIR band in 2016.

The recollision probability is widely used in the VNIR band, but no research has been done to evaluate its behavior in the TIR band. We statistically analyze the recollision probability in the TIR band using a Monte Carlo (MC) model and the input of a measured leaf spectrum in the HiWATER experiment [34], as shown in Fig. 1 (assuming the soil reflectance is equal to 0 to concern the collisions between leaves). There are two main differences between the radiative transfer processes in the VNIR and TIR bands. The first is that the reflectance in the TIR band is obviously lower than that of VNIR band, while the other difference is that the transmittance in the TIR band is equal to 0 , which means that the photons cannot be transmitted through a leaf in the TIR band.

Fig. 2 shows the MC-simulated recollision probability with different wavelengths, with an LAI equal to 3 and with a spherical leaf angle distribution (LAD). The phenomenon of spectral invariant is also observed in the TIR band when the number of photons is greater than $10^{6}$.

The interception $\left(i_{0}\right)$ of the first collision indicates the interception possibility by the canopy in the incident direction, which can be calculated using (3). There are two possibilities after the first collision between the photon and leaf: absorption or reflection. The reflected photon may escape from the canopy, or collide with other leaves again. If we can calculate the upward escape probability $e_{u}$ and the downward escape probability $e_{d}$, the $p$ value can be obtained using (4). Here, $i_{0}$ stands for the directional interception fraction, $\theta$ is the VZA,

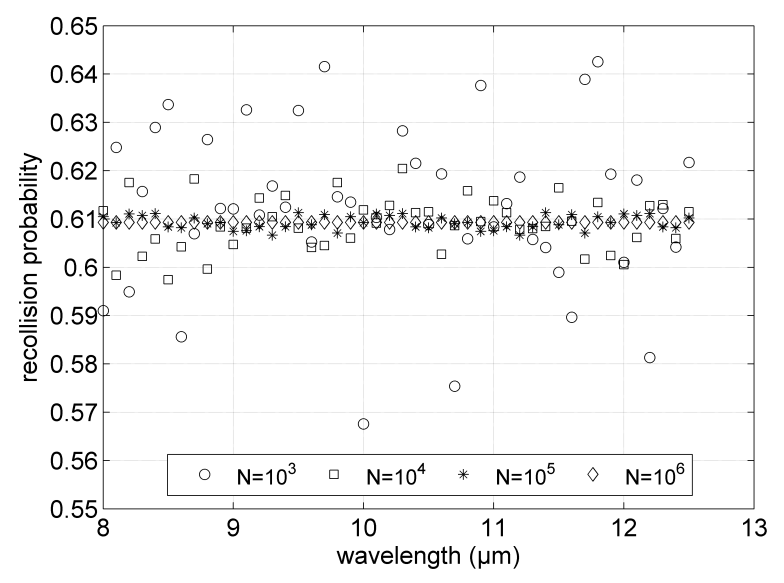

Fig. 2. Recollision probability with different numbers of photons $N$.

LAI is the leaf area index, and $G$ is the mean projection of the unit leaf area on a plane normal to a direction. It can be determined by the LAD and VZA

$$
\begin{aligned}
i_{0} & =1-e^{-G(\theta) \mathrm{LAI} / \cos \theta} \\
p & =1-e_{u}-e_{d} .
\end{aligned}
$$

Taking a thin layer as an example, four probabilities are needed to calculate the upward escape probability of this layer, including the probability of the photon reaching this layer, the probability of interception of this thin layer in the view direction, the upward reflected probability, and the upward hemispherical gap probability. $e_{u}$ can be obtained through integrating the entire canopy height, as shown in the following equation:

$$
\begin{aligned}
e_{u}=\frac{1}{i_{0}} \cdot \int_{0}^{H}\left[e^{-G(\theta) u_{l} h / \cos \theta} \cdot \frac{G(\theta) u_{l} \mathrm{dh}}{\cos \theta} \cdot a_{u}\right. \\
\left.\cdot 2 \int_{0}^{\pi / 2} e^{-G(\theta) u_{l} h / \cos \theta} \cos \theta \sin \theta d \theta\right]
\end{aligned}
$$

where $H$ is the canopy height, $h$ is the height of a certain layer, dh is the thickness of the layer, $u_{l}$ is the leaf area density which is defined as the total one-sided leaf area of photosynthetic tissue per unit canopy volume, $a_{u}$ is the probability of a photon moving upward after being scattered by a leaf. Similarly, $e_{d}$ can also be calculated using (6), where $a_{d}$ is the probability of a photon moving downward after being scattered by a leaf

$$
\begin{aligned}
e_{d}=\frac{1}{i_{0}} \cdot \int_{0}^{H}\left[e^{-G(\theta) u_{l} h / \cos \theta} \frac{G(\theta) u_{l} \mathrm{dh}}{\cos \theta} \cdot a_{d}\right. \\
\left.\cdot 2 \int_{0}^{\pi / 2} e^{-G(\theta) u_{l}(H-h) / \cos \theta} \cos \theta \sin \theta d \theta\right] .
\end{aligned}
$$

Equations (5) and (6) are approximations because the vegetation layer is not lambertian. However, they prove to be good approximations if the LAD is spherical with lambertian leaves. In that case, $a_{u}$ and $a_{d}$ can be considered equal to 0.5 . Below, this simplifying hypothesis is always assumed if not specifically stated. 


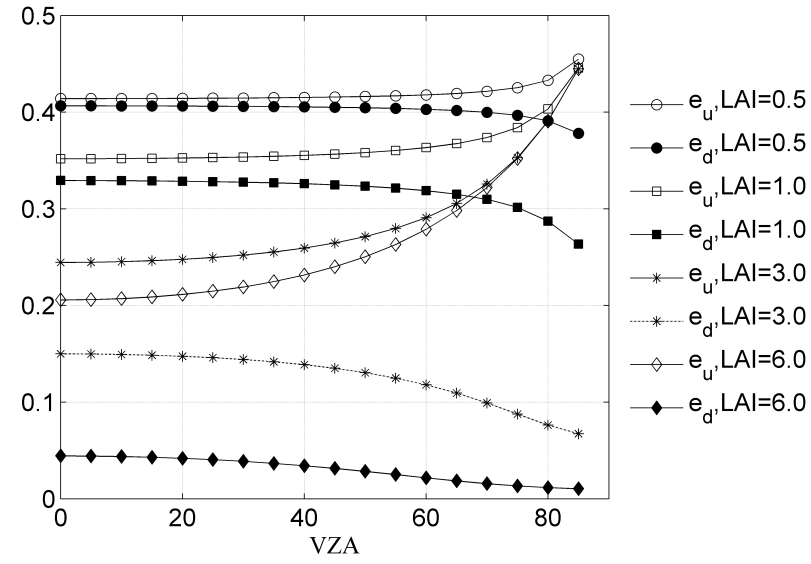

Fig. 3. $e_{u}$ and $e_{d}$ with different LAIs and VZAs.

As shown in Figs. 3 and $4, e_{u}, e_{d}$, and $p$ are angledependent. Furthermore, $e_{u}$ decreases with LAI and increases with VZA. The bigger the LAI, the steeper its increase with VZA. $e_{d}$ also drops with LAI; however, contrary to $e_{u}$, it decreases slightly with VZA. Therefore, the $p$ value increases with LAI because it is equal to $1-e_{u}-e_{d}$. The $p$ value also drops slightly with VZA, as shown in Fig. 4.

\section{Other Important Parameters Based on Spectral Invariants}

After the first collision, the unabsorbed photons will be scattered. Part of the scattered photons will escape from the canopy top or bottom, and others will recollide with the leaves again and again. Considering this multiscattering effect, and further assuming that the $p$ remains constant in successive interactions, the forward (backward) diffuse reflectance of the canopy $r_{c 1}^{*}\left(r_{c 2}^{*}\right)$ can be calculated using the following equations:

$$
\begin{aligned}
& r_{c 1}^{*}=w \cdot e_{d}+w \cdot p \cdot w \cdot e_{d}+w^{2} \cdot p^{2} \cdot w \cdot e_{d}+\cdots=\frac{w \cdot e_{d}}{1-w \cdot p} \\
& r_{c 2}^{*}=w \cdot e_{u}+w \cdot p \cdot w \cdot e_{u}+w^{2} \cdot p^{2} \cdot w \cdot e_{u}+\cdots=\frac{w \cdot e_{u}}{1-w \cdot p}
\end{aligned}
$$

where $w$ is the single-scattering albedo in the VNIR and is equal to the sum of the leaf reflectance and leaf transmittance. However, the leaf transmittance is 0 in the infrared band, such that $w$ is equal to the leaf reflectance value, which can be obtained through (9), where $\varepsilon_{v}$ is the leaf emissivity

$$
w=1-\varepsilon_{v} .
$$

For a clumped canopy, the directional gap fraction $\left(b_{0}\right)$ can be calculated using (10), which was proposed by Nilson [35]. $\Omega$ is the clumping index. This parameter is equal to 1 for a homogeneous canopy. Note that the sum of $b_{0}$ and $i_{0}$ is equal to 1

$$
b_{0}=1-i_{0}=e^{-\Omega G(\theta) \mathrm{LAI} / \cos \theta} .
$$

The hemispherical average intercept probability of the canopy $\left(i_{0}^{\prime}\right)$ can be obtained by integrating the directional

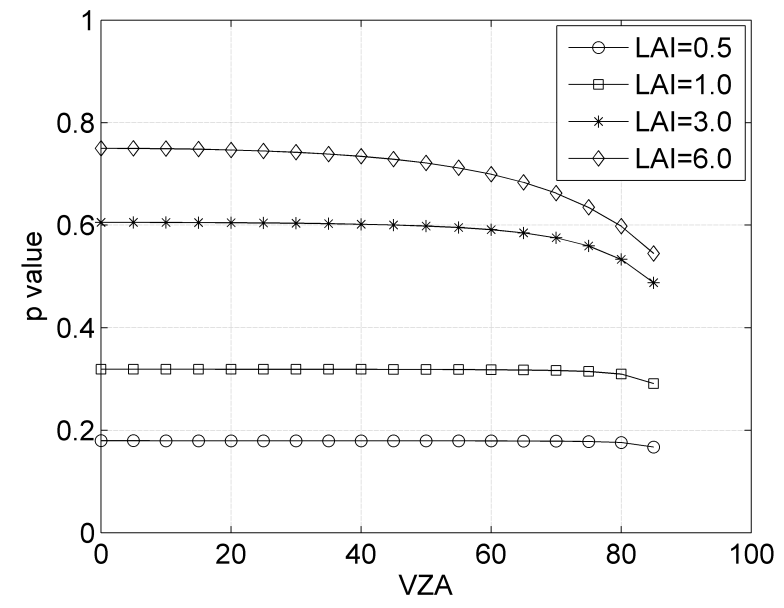

Fig. 4. $\quad p$ value with different LAIs and VZAs.

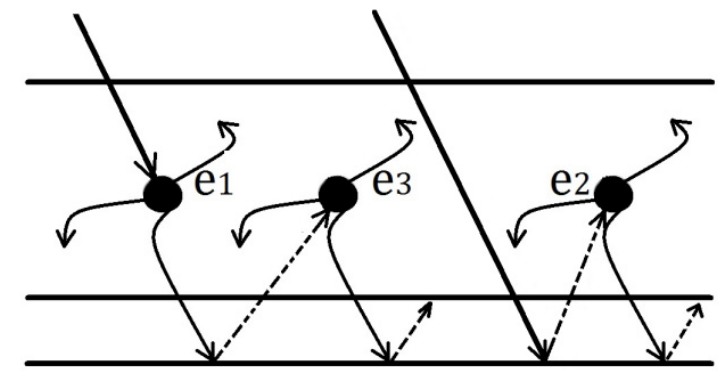

Fig. 5. Illustration of the composition of the vegetation absorption.

intercept probability $i_{0}$, as shown in (11). Fan et al. [29] proposed an empirical exponential function for the spherical LAD

$$
i_{0}^{\prime}=2 \int_{0}^{\frac{\pi}{2}}\left(1-e^{-\Omega G(\theta) \mathrm{LAI} / \cos \theta}\right) \cos \theta \sin \theta d \theta .
$$

\section{Basic Model}

Based on Kirchhoff's law, the directional emissivity is equal to the directional absorption of the canopy, as shown in (12). Furthermore, the directional absorption is the sum of the vegetation directional absorptance $a v(\theta)$ and the background directional absorptance as $(\theta)$

$$
e(\theta)=a(\theta)=a v(\theta)+a s(\theta) .
$$

The $a v(\theta)$ includes three parts, as shown in Fig. 5: the direct absorption of radiation by the vegetation $(e 1)$, the radiation that reaches the background and is reflected by the background before being absorbed by the vegetation $(e 2)$, and the radiation that reaches the background after one or more scattering events within the canopy and is reflected by the background before being absorbed by the vegetation (e3).

In addition, the remaining energy after $e 2$ and $e 3$ absorption will reach the background again and be reflected by the background before being absorbed by the vegetation. The multiple scatterings between the vegetation and background can be calculated by the diffuse reflectance of the vegetation 


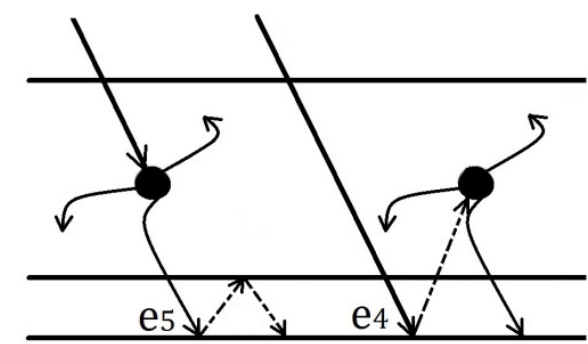

Fig. 6. Illustration of the composition of background absorption.

canopy $r_{c}^{*}$ [see (7) and (8)] and the hemispherical average intercept probability of the canopy $i_{0}^{\prime}$ [see (11)]. Considering the multiple collisions between the components and photons, the vegetation absorptance can be deduced as follows:

$$
\begin{aligned}
\operatorname{av}(\theta)= & e 1+e 2+e 3=\frac{i_{0} \varepsilon_{v}}{1-p\left(1-\varepsilon_{v}\right)} \\
& +\frac{\left(1-i_{0}\right)\left(1-\varepsilon_{s}\right) i_{0}^{\prime} \cdot \frac{\varepsilon_{v}}{1-p\left(1-\varepsilon_{v}\right)}}{1-r_{c 2}^{*}\left(1-\varepsilon_{s}\right) i_{0}^{\prime}} \\
& +\frac{i_{0} r_{c 1}^{*}\left(1-\varepsilon_{s}\right) i_{0}^{\prime} \cdot \frac{\varepsilon_{v}}{1-p\left(1-\varepsilon_{v}\right)}}{1-r_{c 2}^{*}\left(1-\varepsilon_{s}\right) i_{0}^{\prime}}
\end{aligned}
$$

where $p, i_{0}$, and $i_{0}^{\prime}$ can be determined by LAI, LAD, and $\mathrm{VZA}$, and $\varepsilon_{s}$ and $\varepsilon_{v}$ are the background emissivity and leaf emissivity. We assume that the component emissivities are lambertian.

As shown in Fig. 6, as $(\theta)$ includes two parts: the direct absorption of radiation by the background (e4), and the absorption by the background after one or more scattering events within the canopy (e5). In addition, the remaining energy after $e 4$ and $e 5$ absorptions will reach the vegetation and diffuse reflected by the vegetation before being absorbed by the background again. The equation of $a s(\theta)$, which considers the multiple scatterings between the vegetation and background, is shown as follows:

$$
a s(\theta)=e 4+e 5=\frac{\left(1-i_{0}\right) \varepsilon_{s}}{1-\left(1-\varepsilon_{s}\right) i_{0}^{\prime} r_{c 2}^{*}}+\frac{i_{0} r_{c 1}^{*} \varepsilon_{s}}{1-\left(1-\varepsilon_{s}\right) i_{0}^{\prime} r_{c 2}^{*}} .
$$

\section{SEnsitivity Analysis}

\section{A. Sensitivity Analysis of the Basic Model}

The input parameters are listed in Table I. Four LAIs, 18 VZAs, one leaf emissivity, and one soil emissivity constitute 72 samples. The LAD of these samples is spherical as introduced above. The canopy emissivity and the five components change with VZA and LAI, as shown in Fig. 7.

For small LAIs (for example, LAI $=0.5,1$ ), the canopy emissivities clearly increase with VZA because more leaves can be observed in the oblique view, and the leaf emissivity is larger than the soil emissivity. Although $e 2$ and $e 4$ decrease with VZA, the growth of $e 1$ exceeds their combined decreases.

For large LAIs (for example, LAI $=3,6$ ), the canopy emissivities decrease with VZA, as shown in Fig. 7, which
TABLE I

Model Key InPUTS

\begin{tabular}{cc}
\hline Parameter & Value \\
\hline Leaf emissivity & 0.98 \\
Soil emissivity & 0.94 \\
LAI & $0.5,1.0,3.0,6.0$ \\
VZA & $0-85$, step $=5$ \\
\hline
\end{tabular}
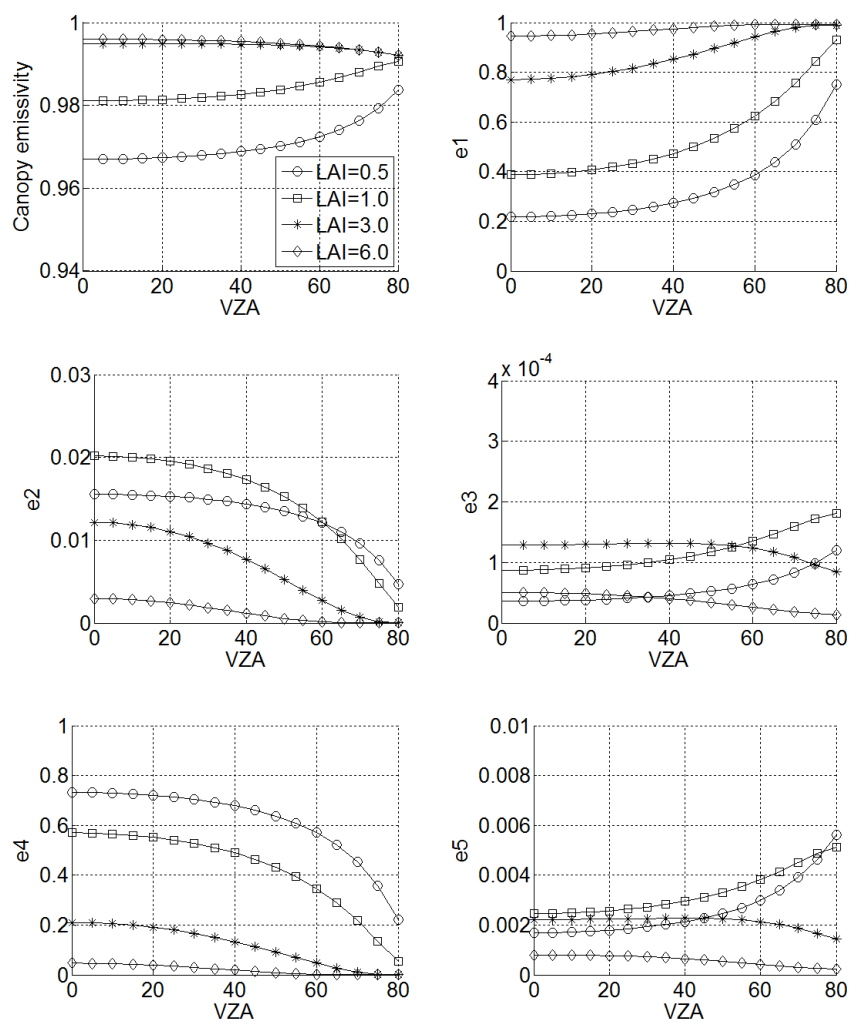

Fig. 7. Canopy emissivity and the five components with VZAs and LAIs.

is contrary to the trend of small LAIs. Under these circumstances, the observed leaf coverage in the nadir direction is already very large, and the growth of $e 1$ is less than the combined decline of $e 2$ and $e 4$. Furthermore, Fig. 7 shows that a dense vegetated canopy has a low angular variation of emissivity, almost corresponding to a lambertian surface.

The trends of $e 1$ and $e 4$ are easy to understand and are controlled by the gap fraction. $e 3$ is small enough to be ignored in all cases. $e 5$ can be ignored for small VZAs, but should be considered for VZAs larger than $40^{\circ} . e 2$ always decreases with VZA because of the smaller gap fraction caused by the larger view angle. However, there is a cross point for the line of $\mathrm{LAI}=0.5$ and the line of $\mathrm{LAI}=1$ because $e 2$ is proportional to the hemispherical average intercept probability $\left(i_{0}^{\prime}\right)$ and the directional gap fraction $\left(1-i_{0}\right)$, while $1-i_{0}$ is inversely proportional to LAI, but $i_{0}^{\prime}$ is proportional to LAI. When LAI increases from 0.5 to 1 , for small VZAs, the growth of $i_{0}^{\prime}$ 


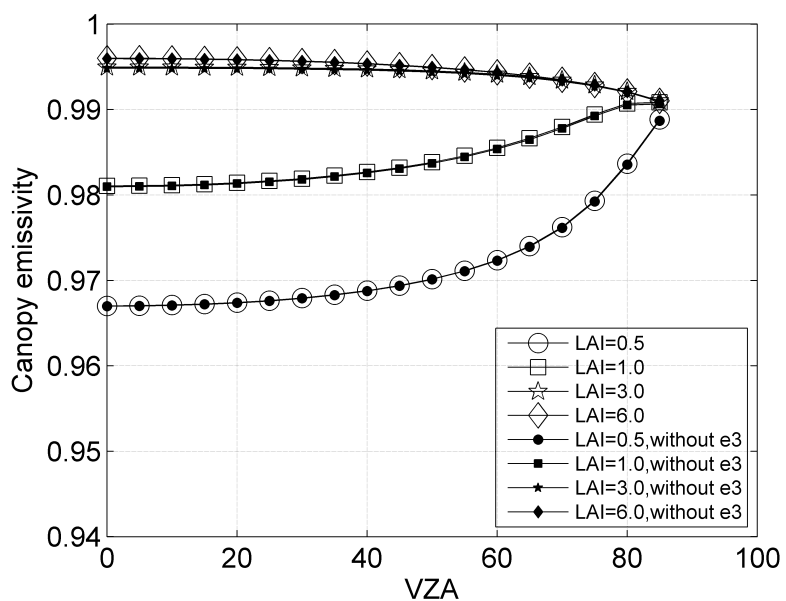

Fig. 8. Comparison of the canopy emissivities with $e 3$ and without $e 3$.

is greater than the decline of $1-i_{0}$. Therefore, the line of $\mathrm{LAI}=1$ spans up to the line of $\mathrm{LAI}=0.5$. For large VZAs, the growth of $i_{0}^{\prime}$ is smaller than the decline of $1-i_{0}$. Thus, the trend is reversed, leading to an intersection of the two lines.

\section{B. Basic Model Simplification}

Four steps of simplification of the basic model are proposed based on the sensitivity analysis in Section III-A.

Step 1: If we ignore the contribution of $e 3$, the bias will be less than 0.0002 , while (13) will be more concise. The comparison between $(e 1+e 2+e 3+e 4+e 5)$ and $(e 1+e 2+e 4+e 5)$ is shown in Fig. 8. e3 can be omitted under this circumstance.

Step 2: Furthermore, if we ignore the multiple scatterings between the vegetation and background, the directional emissivity can be calculated using the following equation. The canopy emissivity will drop slightly, as shown in Fig. 9

$$
\begin{aligned}
e= & \frac{i_{0} \varepsilon_{v}}{1-p\left(1-\varepsilon_{v}\right)}+\left(1-i_{0}\right)\left(1-\varepsilon_{s}\right) i_{0}^{\prime} \\
& \times \frac{\varepsilon_{v}}{1-p\left(1-\varepsilon_{v}\right)}+\left(1-i_{0}\right) \varepsilon_{s}+i_{0} r_{c 1}^{*} \varepsilon_{s} .
\end{aligned}
$$

Step 3: Based on (15), we further ignore the multiscattering within the vegetation of $e 2$ and $e 5$, as shown in (16). Fig. 10 shows that the canopy emissivity will drop very slightly. Therefore, the contribution of multiscattering within $e 2$ and $e 5$ can also be ignored

$$
\begin{array}{r}
e=\frac{i_{0} \varepsilon_{v}}{1-p\left(1-\varepsilon_{v}\right)}+\left(1-i_{0}\right)\left(1-\varepsilon_{s}\right) i_{0}^{\prime} \varepsilon_{v} \\
+\left(1-i_{0}\right) \varepsilon_{s}+i_{0}\left(1-\varepsilon_{v}\right) e_{d} \varepsilon_{s} .
\end{array}
$$

Step 4: However, if the multiscattering in $e 1$ is ignored, as is done for $e 2$ and $e 5$, the canopy emissivity will decrease rapidly, as shown in Fig. 11, especially for large LAIs. Thus, it should be considered.

The drop is less than 0.006 for small LAIs, as shown in Fig. 12. The multiscattering within $e 1$ is inversely proportional to $1-p\left(1-\varepsilon_{v}\right)$, while $p$ is proportional to LAI.

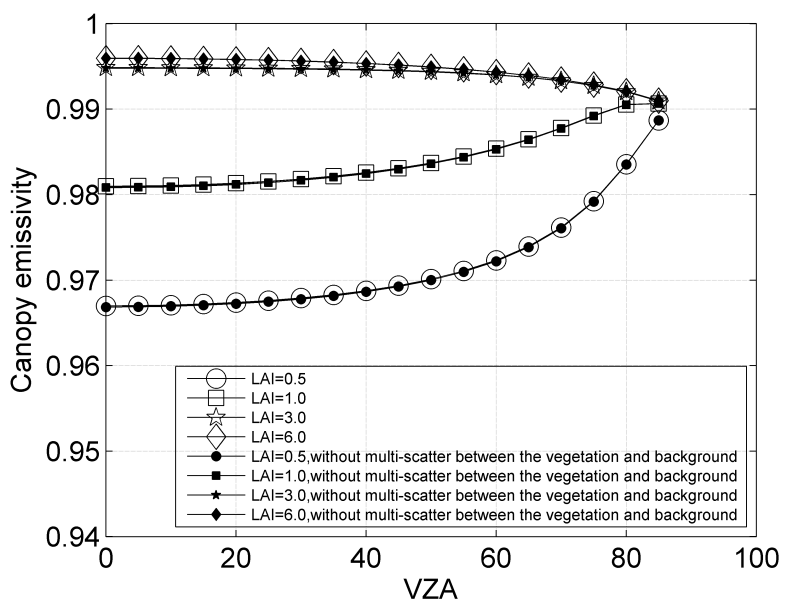

Fig. 9. Comparison of the canopy emissivities with and without multiscattering between the vegetation and background.

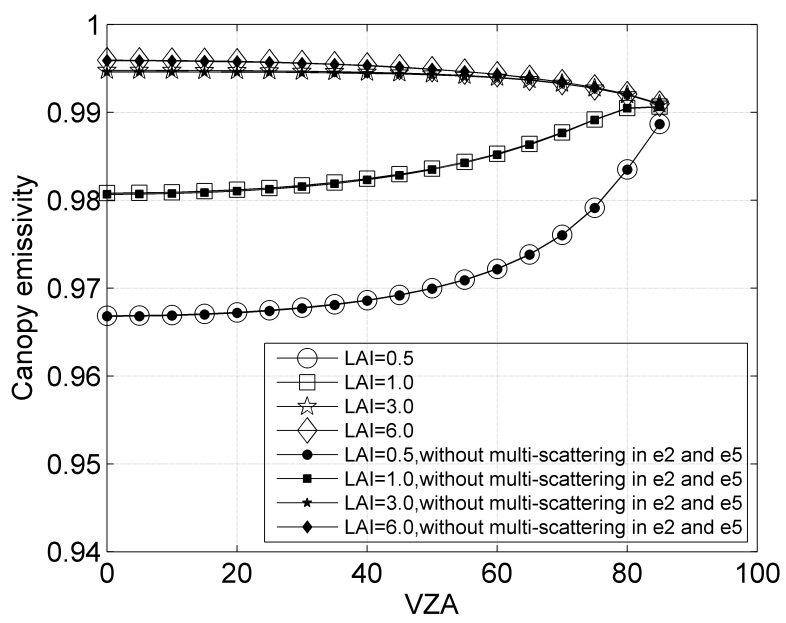

Fig. 10. Comparison of the canopy emissivities with and without the multiscattering contribution of $e 2$ and $e 5$.

Therefore, a bigger LAI results in a larger multiscattering effect. The difference is larger than 0.01 for large LAIs, making this step of simplification unacceptable, as shown in Fig. 12.

Furthermore, we try to analyze the order of the recollisions that should be considered in the term of $e$. Fig. 13 shows the canopy emissivity that considers only the first and second terms of the geometric series of $e 1$ [see (17)]. The outputs are very close to the result of (16), indicating that the third-order term and beyond can be omitted for simplification because the leaf reflectance is very small (for example, 0.02 in the 72 samples) in the TIR band

$$
\begin{aligned}
e=i_{0} \varepsilon_{v}+i_{0} \varepsilon_{v} p\left(1-\varepsilon_{v}\right) & +\left(1-i_{0}\right)\left(1-\varepsilon_{s}\right) i_{0}^{\prime} \varepsilon_{v} \\
+ & \left(1-i_{0}\right) \varepsilon_{s}+i_{0}\left(1-\varepsilon_{v}\right) e_{d} \varepsilon_{s} .
\end{aligned}
$$

Above all, from the perspective of absorption, the final proposed simplified analytical equation of the canopy directional emissivity can be expressed as (17), wherein only the first and second collisions between the leaf or soil and the photons are considered. This simplification with less complexity and 


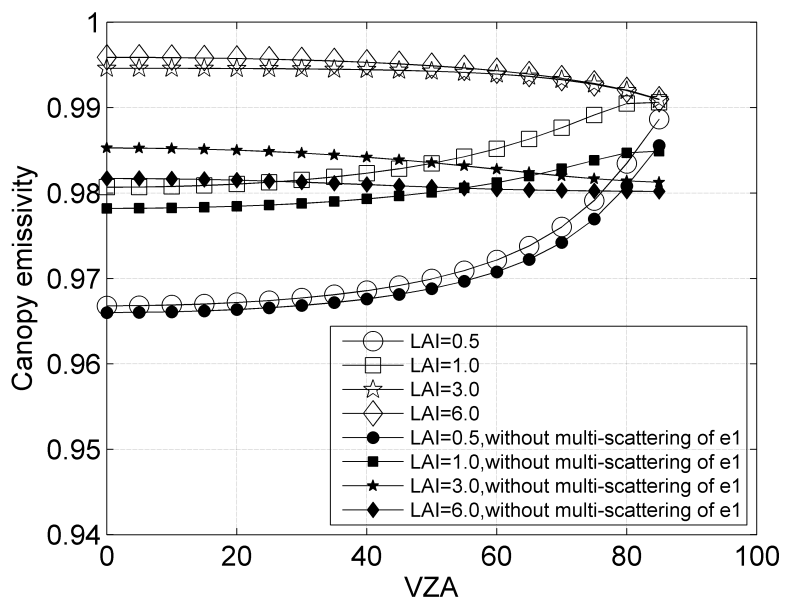

Fig. 11. Comparison of the canopy emissivities with and without the multiscattering in $e 1$.

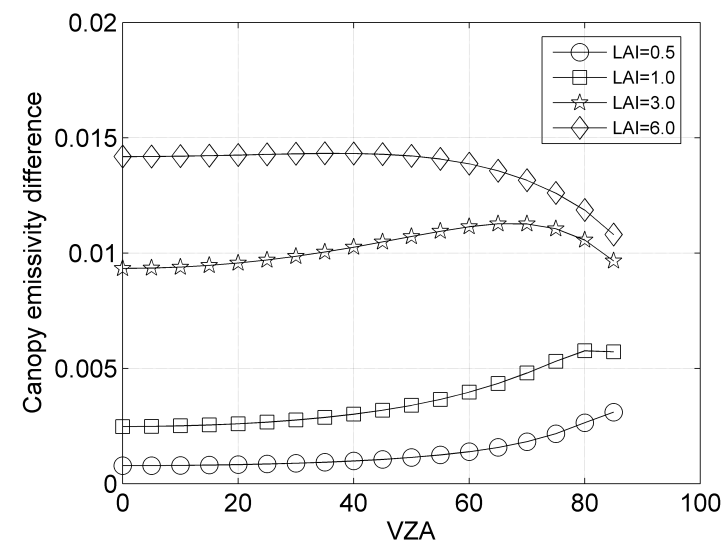

Fig. 12. Canopy emissivity difference with/without the multiscattering in $e 1$.

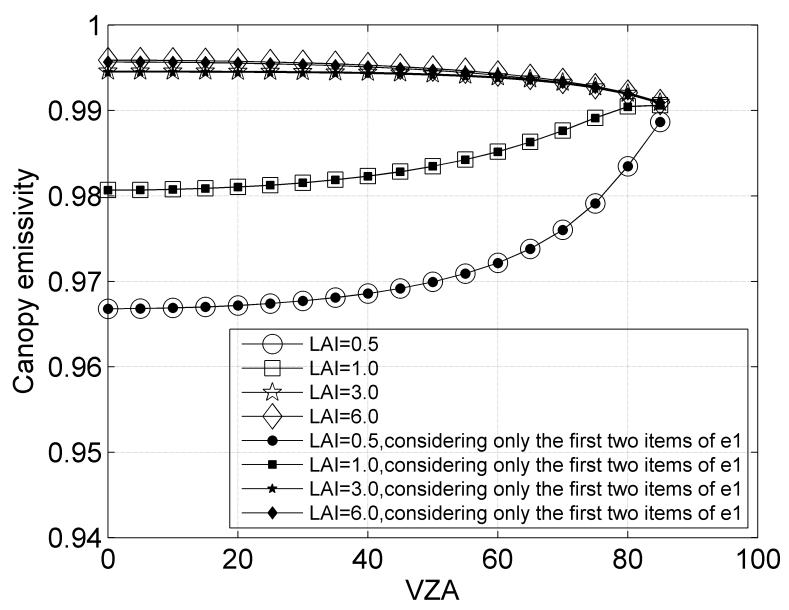

Fig. 13. Comparison of the canopy emissivities of (16) and (17).

almost the same accuracy will be benefit to the land surface component temperature inversion and the scaling of LST. From the perspective of active emissions of canopy components in the TIR band, this simplification means that only the direct emission (first collision) and the single-scattering effect

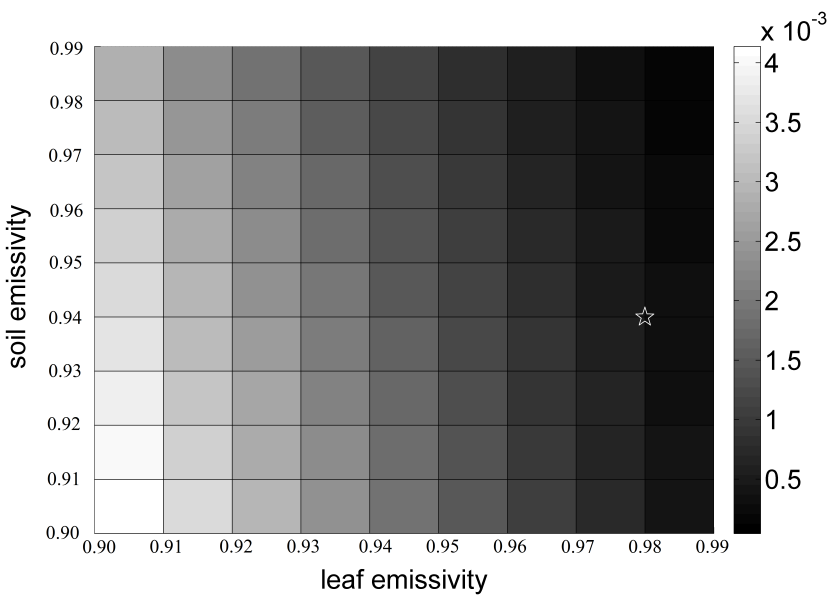

Fig. 14. Mean differences between the full [see (12)] and simplified [see (17)] canopy emissivity for different leaf and soil emissivity values.

(second collision) need to be considered, which will be discussed in Section VI.

\section{Sensitivity Analysis of the Simplified Model}

The simplification in this section is deduced under the condition of unique leaf emissivity (0.98) and soil emissivity (0.94). What will occur if the input emissivities change? We calculated the mean differences of 72 samples (comprising 4 LAIs and 18 VZAs, as shown in Table I) with different component emissivity combinations (growing up to 0.99 from 0.90 with a step size of 0.01 , respectively). It can be observed from Fig. 14 that the difference between (12) and (17) will increase with the decrease of leaf emissivity or the drop of soil emissivity.

The difference will be up to 0.004 when the leaf and soil emissivities are equal to 0.90 and will be no more than 0.002 when the leaf emissivity is equal to 0.98 and the soil emissivity is equal to 0.94 , as shown by the star in Fig. 14 . The difference caused by model simplification is more sensitive to the leaf emissivity than to soil emissivity. If the leaf emissivity is over 0.94, as shown in Fig. 14, the difference is less than 0.0025 , which means that the simplification is acceptable. We statistically analyze the spectral library of UCSB, and find that $0.94(0.90)$ can be regarded as the boundary value of leaf (soil) emissivity. Therefore, the simplification of the basic model is practical for application.

Furthermore, we can evaluate the contributions of the differences between (12) and (17) in detail under the help of the analytical equations of CE-P. There are four steps of simplification, including ignoring $e 3$, the multiple scatterings between the vegetation and background in $e 2, e 4$, and $e 5$, the multiscattering within the vegetation of $e 2$ and $e 5$, and the third-order and above terms of $e 1$. The contributions of these steps (see Figs. 15-18) can be calculated separately because CE-P is an analytical model.

Overall, the patterns in Figs. 15-17 are similar to that of Fig. 14. When the leaf or soil emissivity is smaller, the difference is larger. Nevertheless, the contribution of the fourth step of the simplification in Fig. 18 is related only to the leaf 


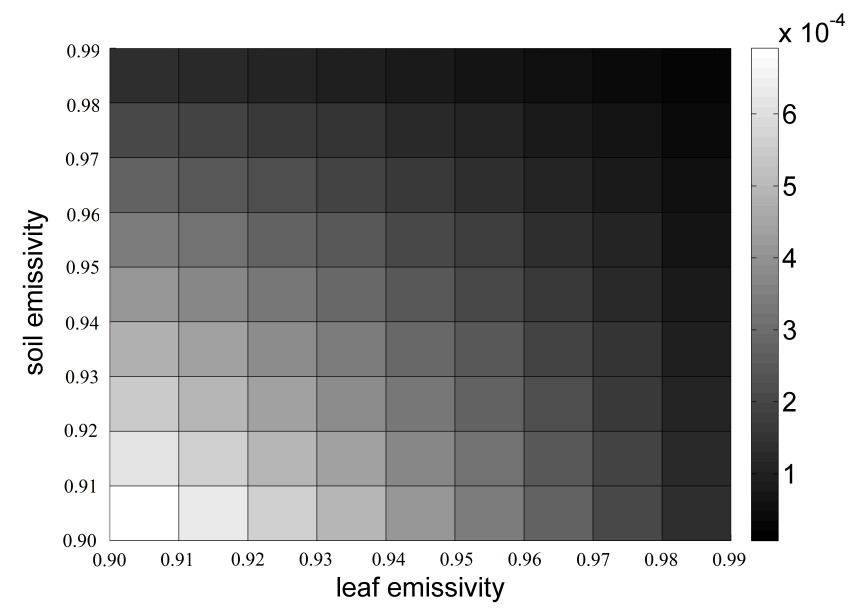

Fig. 15. Mean difference caused by the first step of the simplification (neglecting the contribution of $e 3$ ) for different leaf and soil emissivity values.

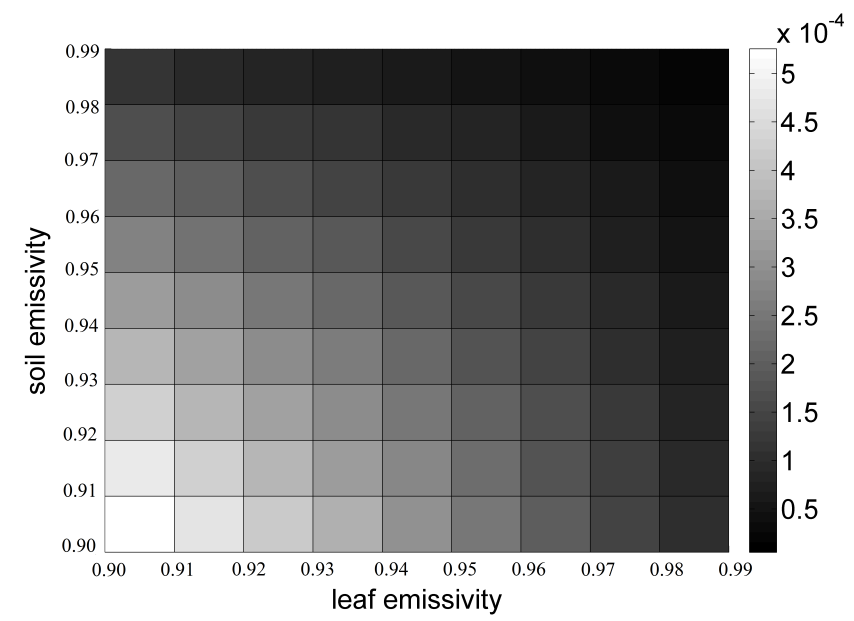

Fig. 16. Mean difference caused by the second step of the simplification (neglecting the multiscattering between the vegetation and background in $e 2$, $e 4$, and $e 5$ ) for different leaf and soil emissivity values.

emissivity: when the leaf emissivity is smaller, the difference is larger. This difference can be as high as 0.002 when the leaf emissivity is 0.90 , which means the third-order and beyond terms of $e 1$ should be considered in this case. The difference will be less than 0.001 when the leaf emissivity is over 0.94 . Thus, the simplified model can achieve a high accuracy in practice.

\section{Cross Validation}

The 4SAIL and DART are the most accurate RTM and ray tracing model for a homogeneous canopy in the TIR band, respectively. They can accurately produce the canopy emissivity distribution, which is suitable for the cross validation of the new model. During the cross validation, the leaf emissivity and soil emissivity are set as 0.98 and 0.94 , as shown in Table I, but the LAI and VZA will be interpolated to give more detailed results. In this section, the simplified model (CE-P) is selected to do cross validation, and the details are introduced as follows.

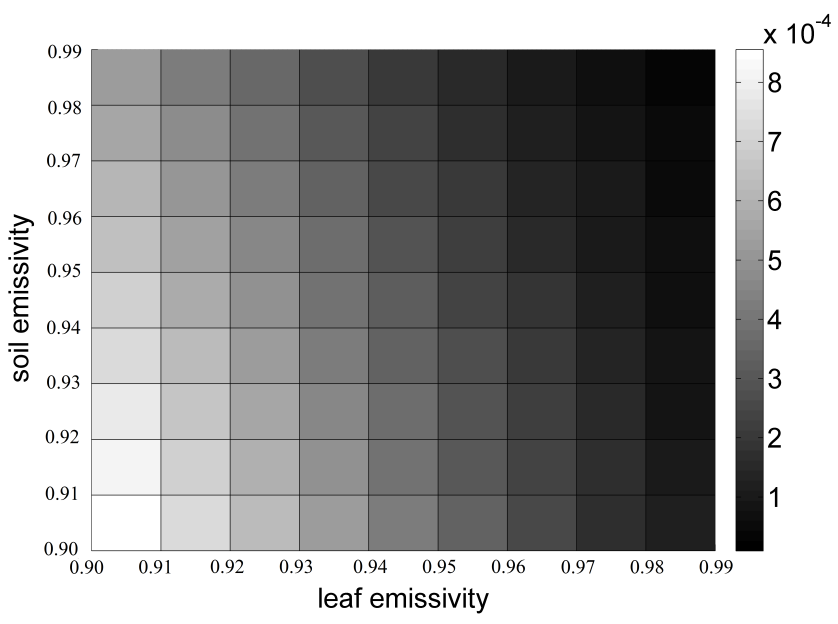

Fig. 17. Mean difference caused by the third step of the simplification (neglecting the multiscattering within the vegetation of $e 2$ and $e 5$ ) for different leaf and soil emissivity values.

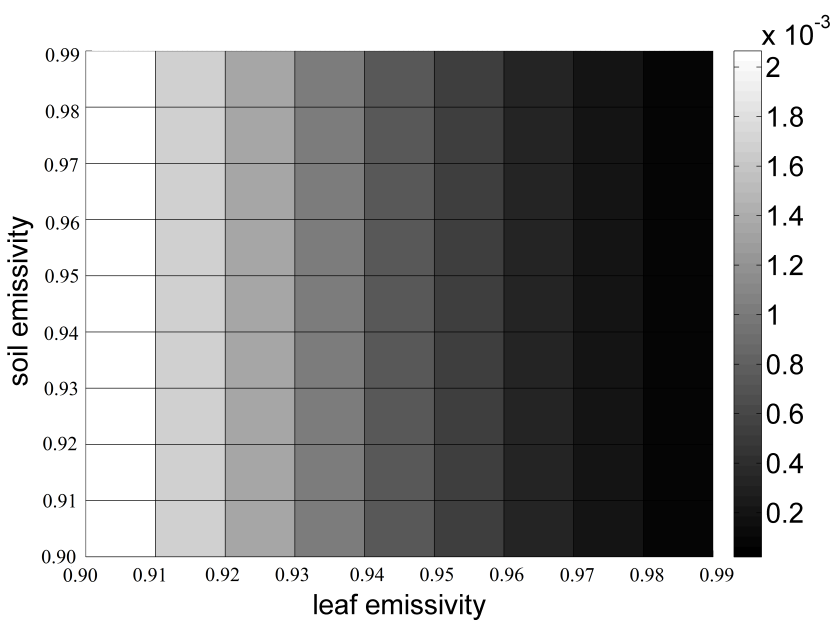

Fig. 18. Mean difference caused by the fourth step of the simplification (neglecting the third-order and beyond terms of $e 1$ ) for different leaf and soil emissivity values.

\section{A. Validation Using the 4SAIL Model}

The SAIL model is a four-steam RTM proposed by Verhoef et al. [36]. In 2007, the SAIL model was extended to the TIR domain with the same model framework but considering the temperature differences between the sunlit and shaded leaves/soils. This extension enables the simulation of the TIR directionality with considering the multiple scatterings inside the vegetation canopy. One advantage of the 4SAIL is its detailed estimation of the multiple scattering effects due to the detailed radiative transfer theory. If we set the temperatures of the four components to the same value, then we can derive the canopy emissivity as the ratio of the directional canopy radiance and the blackbody radiance at the same temperature, the same component emissivities, and canopy structures. Setting the same component temperatures can avoid the definitional differences of the $r$-emissivity of the CE-P and the $e$-emissivity of the 4SAIL outputs [37]. 


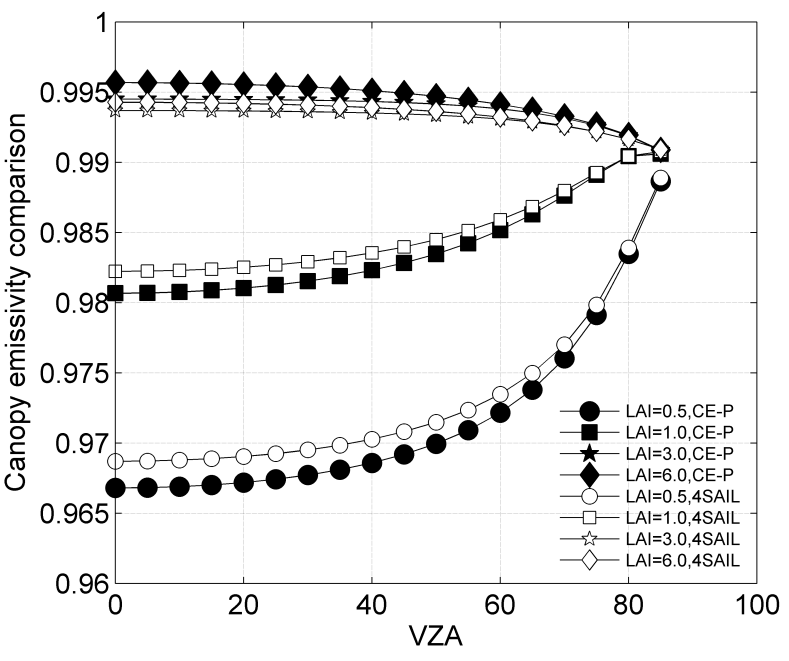

Fig. 19. Comparison between CE-P and 4SAIL.

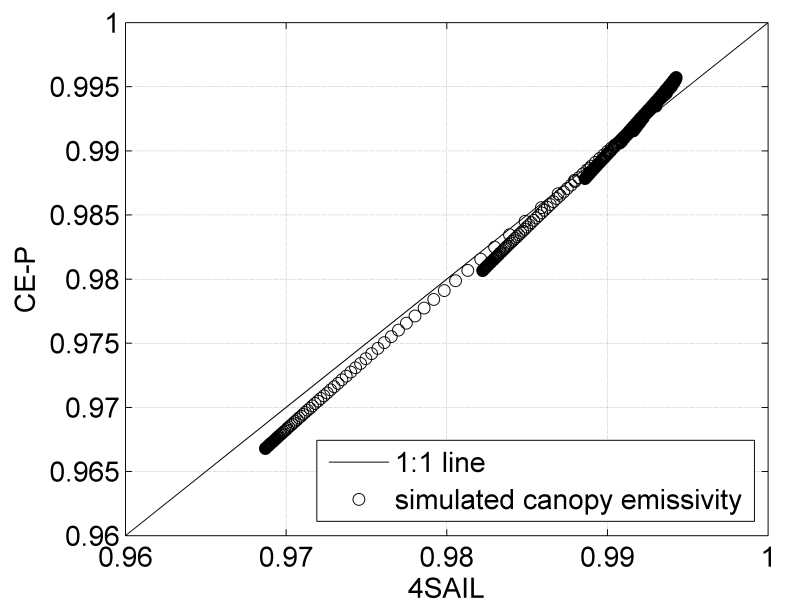

Fig. 20. Scatterplot between CE-P and 4SAIL.

The 4SAIL is an analytical model with differential equations and runs very fast. It is a reliable reference to cross validate our new model. Fig. 19 shows a comparison between the CE-P and 4SAIL models with the same input parameters as in Table I. CE-P is slightly lower than 4SAIL for small LAIs, while the phenomenon is opposite to that of large LAIs. However, the absolute bias between them is less than 0.002 , which means that compared with 4SAIL, CE-P can achieve a very high accuracy.

Furthermore, the LAI in Table I is set to range from 0.5 to 8 at steps equal to 0.5 , and the VZA in Table I is set to range from 0 to 85 at $1^{\circ}$ steps. The comparison results of these 1376 samples are shown in Figs. 20 and 21. It can be seen from Fig. 21 that the absolute differences between 4SAIL and CE-P are less than 0.002 , which is always acceptable because the 4SAIL model can be treated as the relative true value for a homogeneous canopy simulation.

In addition, we simulated the canopy emissivities of these 1376 samples with two other combinations of component emissivities (group $\mathrm{A}, \varepsilon_{v}=0.94$ and $\varepsilon_{s}=0.90$; group B, $\varepsilon_{v}=0.99$ and $\left.\varepsilon_{s}=0.97\right)$ to evaluate the biases of the simplifications of CE-P. When the component emissivities are

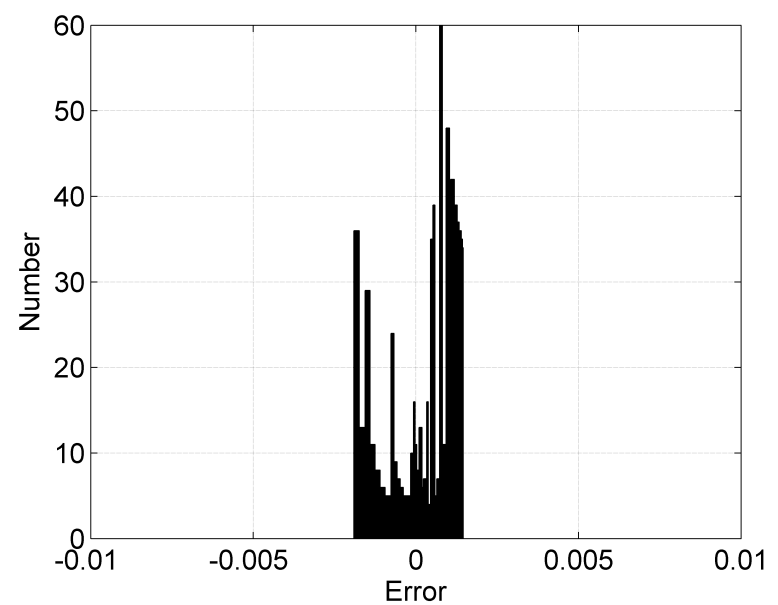

Fig. 21. Directional canopy emissivity error distribution of CE-P when the 4SAIL is regarded as a reference.

smaller, the contribution of the multiple scatterings is greater, which leads to a larger bias of simplified CE-P. We found that the absolute biases between CE-P and 4SAIL are less than 0.003 for group A and less than 0.001 for group B. Their scatterplots and error distributions are omitted for brevity.

\section{B. Validation Using the DART Model}

The DART model is one of the most comprehensive physically based 3-D models simulating the earth-atmosphere radiation interactions from the visible to TIR wavelengths. The DART forward simulations of vegetation reflectance were successfully verified by real measurements and crosscompared with a number of independently designed 3-D reflectance models in the context of the RAdiation transfer Model Intercomparison experiment. The model has been developed since 1992 and was expanded to the TIR band in 2003. The dependence of thermal emissions on temperature and wavelength is modeled with Planck's law. DART output is also widely used as a true value when simulating the brightness temperature distributions over homogeneous and heterogeneous land surfaces in the TIR band.

Fig. 22 shows a comparison between the CE-P and DART models with the same input parameters as in Table I. The absolute bias between them is less than 0.0015 (see Fig. 23), meaning that CE-P can achieve a very high accuracy when compared to DART. The outputs of CE-P are slightly closer to those of DART than those of 4SAIL, as shown in Figs. 21 and 24. Thus, we conclude that CE-P can always precisely simulate the canopy directional emissivity. The slightly difference in Figs. 21 and 24 may be caused by the major differences between 4SAIL and DART. 4SAIL works with four discrete directions (sun, viewing, upward, and downward) whereas DART can work with any number of discrete directions (100 directions in our simulation), 4SAIL solves differential equations whereas DART tracks rays, and 4SAIL defines the LAD with 13 leaf zenith angles whereas DART defines it with 90 leaf zenith steps. 


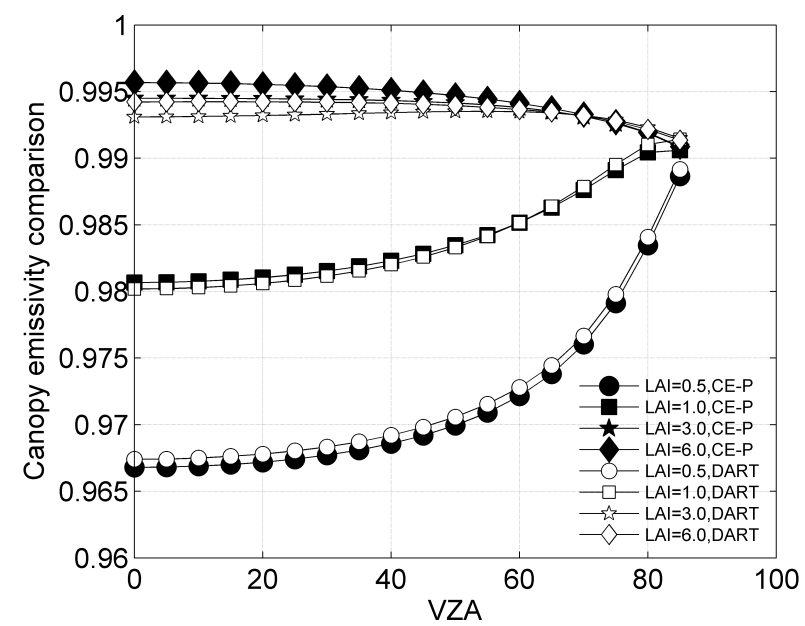

Fig. 22. Comparison between CE-P and DART.

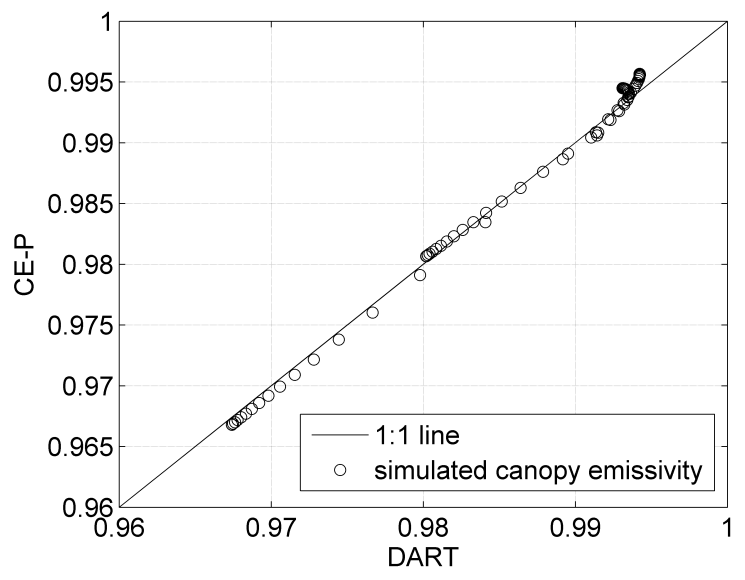

Fig. 23. Scatterplot of CE-P and DART.

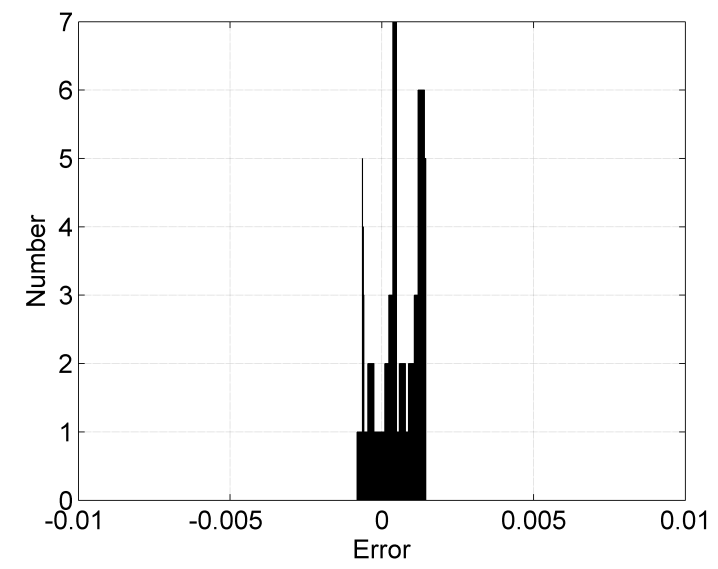

Fig. 24. Directional canopy emissivity error distribution of CE-P when the DART is regarded as a reference.

\section{COMPARISON}

In this section, four widely used analytical canopy emissivity model in the parameter estimation is introduced and compared with CE-P, respectively. In Section V-E, they are compared with CE-P together. The CE-P is seemed as the

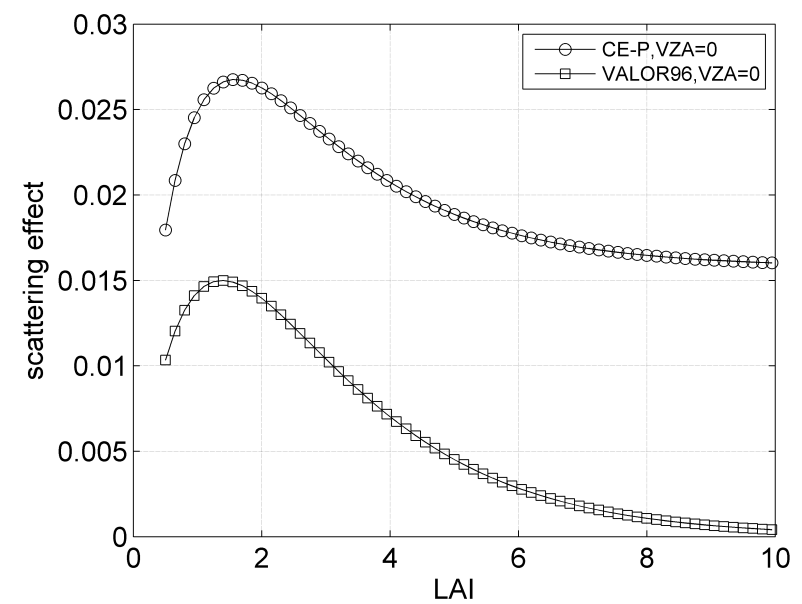

Fig. 25. Comparison between CE-P and VALOR96.

reference to evaluate other models on the basis of validation results with 4SAIL and DART in Section IV.

\section{A. Comparison With VALOR96}

Valor and Caselles [38] proposed a canopy emissivity model for the row-planted landscape using three view factors in 1996 (hereafter, referred to as VALOR96). They further gave an operational equation [see (18)] to calculate the scattering effect based on the proposed canopy emissivity model and to address different land surface types that consisted of leaves and soil. This algorithm was called the DNVI-emissivity method which is the most widely used one for LST inversion [39]

$$
d \varepsilon=4\langle d \varepsilon\rangle i_{0}\left(1-i_{0}\right) .
$$

The $\langle d \varepsilon\rangle$ in (18) was suggested to be set as 0.015 . This algorithm has been widely applied over the world to generate global emissivity product. With the help of the analytical expression of our work, we can evaluate the bias of Valor's operational algorithm. As shown in Fig. 25, although the trend of VALOR96 is similar to that of CE-P, VALOR96 clearly underestimates the canopy emissivity, especially for large LAIs. Because large LAIs will lead to the very small gap fractions (for example, approximately 0.0498 when LAI $=6$ and VZA $=0$ ), the small gap fractions will further lead to the extreme case occurs in $(18)\left(1-i_{0} \approx 0\right.$ and $\left.i_{0} \approx 1\right)$. As shown in (17), the second term will become the main contribution of the scattering effect $(d \varepsilon)$ in this case. However, Valor's operational algorithm cannot simulate this term when the gap fraction is very small $(d \varepsilon \approx 0)$, consequently, results in the observed underestimation.

\section{B. Comparison With FR97}

FR97 was proposed based on the parameterization of the Prevot model, which was a probability-based model that computed the solution of the radiative transfer by summing the relative contributions of a large number of vegetation layers using the directional gap fraction. The Prevot model was not as widely validated as the 4SAIL model. The multiple scatterings within the canopy are underestimated in the Prevot model. 


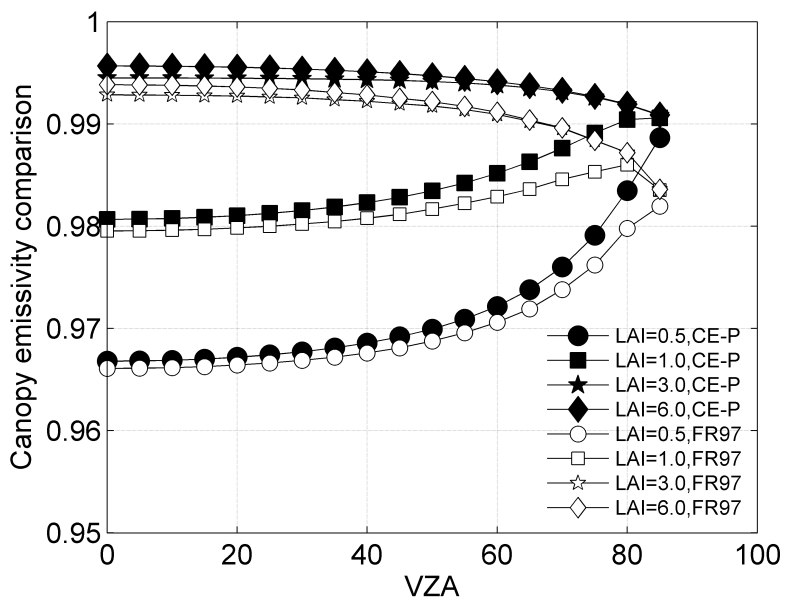

Fig. 26. Comparison between CE-P and FR97.

Therefore, the FR97 always results in underestimations, especially for large VZA values (see Fig. 26) although it behaves very similar to the Prevot model. The equation of FR97 is shown as follows:

$e=1-\left(1-i_{0}\right)\left(1-i_{0}^{\prime}\right)\left(1-\varepsilon_{s}\right)-\alpha\left[1-\left(1-i_{0}\right)\left(1-i_{0}^{\prime}\right)\right]\left(1-\varepsilon_{v}\right)$.

The second term of the right side of (19) is the hemisphericdirectional reflectivity of the soil in the viewing direction, and the third term is the hemispheric-directional reflectivity of the vegetation. $\alpha$ is the cavity effect factor and expresses the part of the incident photons that is reflected by the leaves and then absorbed by the canopy. Therefore, $\alpha$ is used to describe the multiple scatterings inside the canopy. The original values of this parameter for different VZAs were provided by François [18]. The concept of the cavity effect factor is very close to the definition of the $p$ value in our new model. However, $p$ changes with both VZA and LAI.

\section{Comparison With FR02}

Similar to FR97, the model proposed by François [19] in 2002 (FR02) estimated the directional emissivity based on the hemispheric-directional reflectivity but paid more attention to the multiple scatterings at the interface of the soil and vegetation layers, as shown in the following equation:

$$
e=1-i_{0}\left(1-\varepsilon_{v}\right)-\frac{\left(1-i_{0}\right)\left(1-i_{0}^{\prime}\right)\left(1-\varepsilon_{s}\right)}{1-\left(1-\varepsilon_{s}\right) i_{0}^{\prime}\left(1-\varepsilon_{v}\right)} .
$$

The second term of the right-hand side is the reflectivity of the vegetation in the viewing direction, and the third term is the sum of the soil single-scattering reflectivity and multiple scatterings at the interface of the soil and vegetation layers. FR02 places greater weight on multiple scatterings occurring at the interface of the soil and vegetation layers, while FR97 places greater weight on multiple scatterings inside the vegetation. However, our new model can consider all the scattering effects.

FR02 always drastically underestimates (by more than 0.01 on average) the directional canopy emissivities, as shown

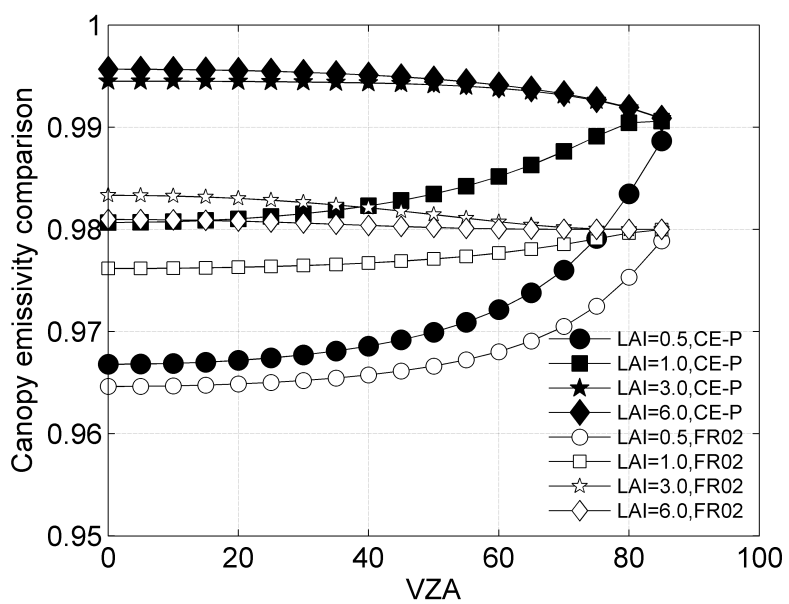

Fig. 27. Comparison between CE-P and FR02.

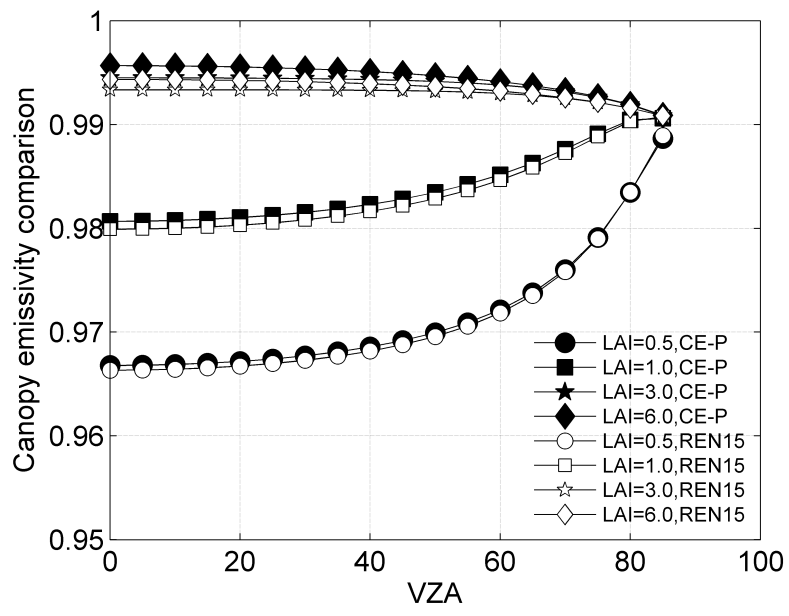

Fig. 28. Comparison between CE-P and REN15.

in Fig. 27. Figs. 26 and 27 show that the multiscattering contribution inside the vegetation is more important than that at the interface of the soil and vegetation layers.

\section{Comparison With REN15}

In order to solve the underestimation of FR97 with large VZAs, Ren et al. [21] recalculated the cavity effect factor using the 4SAIL model as the traditional equation of FR97

$$
\alpha=\left(1-\varepsilon_{\lim }\right) /\left(1-\varepsilon_{v}\right)
$$

where $\varepsilon_{\lim }$ is the limit emissivity that can be obtained with a specified VZA, leaf emissivity, and a given large LAI value (for instance, $\mathrm{LAI}=6$ ). Then, the canopy emissivity becomes closer to the result of 4SAIL, especially when the VZA is large. As shown in Fig. 28, the CE-P result is slightly greater than that of REN15 when the LAI is small. This difference may be due to the logistical problem of (21). Based on the definition of $\alpha$, this parameter can be used only to represent the multiple-scattering effect for dense vegetation (under limit conditions). When using it to simulate the multiscattering for sparse vegetation, the result will face a logistical problem. 


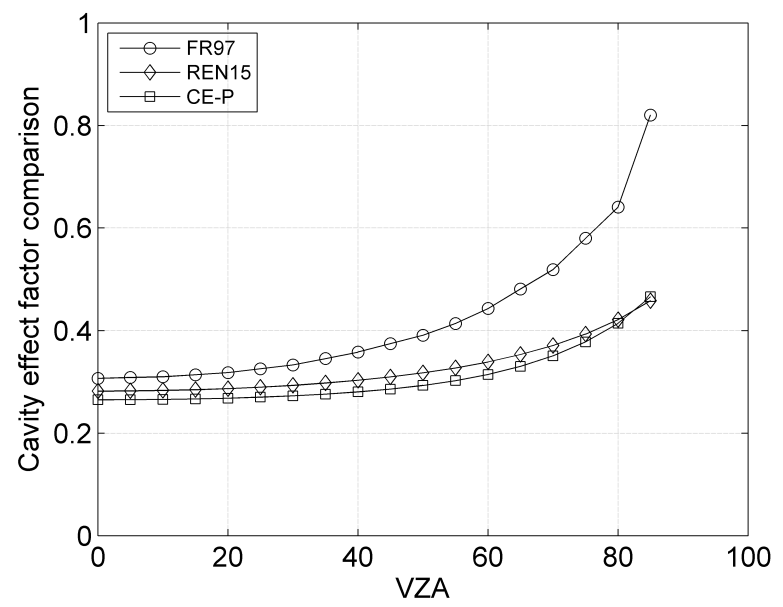

Fig. 29. Cavity effect factor comparison of an LAI equal to 6.
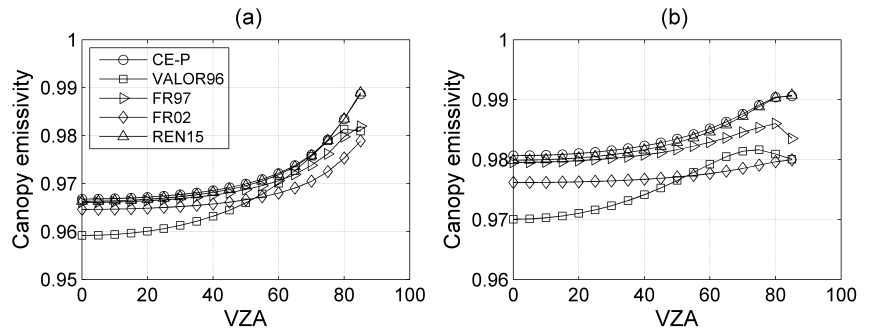

(c)
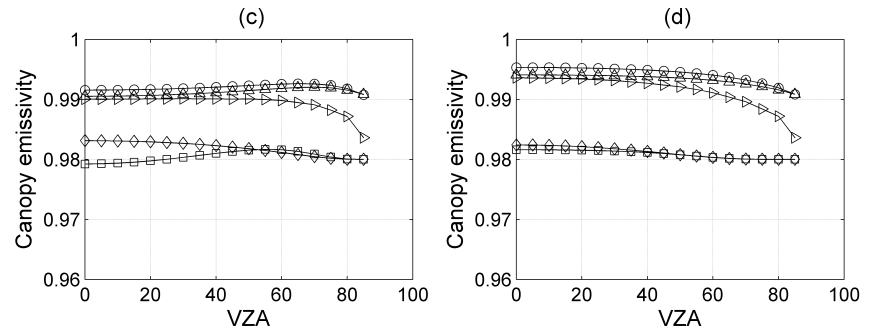

Fig. 30. Canopy emissivity comparison $\left(\varepsilon_{s}=0.94\right.$ and $\left.\varepsilon_{s}=0.98\right)$. (a) $\mathrm{LAI}=0.5$. (b) $\mathrm{LAI}=1$. (c) $\mathrm{LAI}=3$. (d) $\mathrm{LAI}=6$.

The $p$ value proposed in this paper can be applied for canopies with different LAIs and can overcome the logistical problem of REN15. Moreover, our solution can be extended to a heterogeneous canopy. However, both REN15 and CE-P are acceptable for homogeneous canopies because the difference between their results is less than 0.002 .

For very large LAIs, the interception $\left(i_{0}\right)$ is almost equal to 1 and the gap fraction $\left(1-i_{0}\right)$ and downward escape probability $\left(e_{d}\right)$ are almost 0 . Thus, the limit canopy emissivity can be obtained based on CE-P [see (17)] as follows:

$$
\varepsilon_{\lim }=\varepsilon_{v}\left[1+p\left(1-\varepsilon_{v}\right)\right] .
$$

The analytical expression [see (23)] of the cavity effect factor $(\alpha)$ can be obtained based on (21) and (22). Although $\alpha$ is no longer used in CE-P, we can compare the values of FR97, REN15, and CE-P (see Fig. 29). The modified value of the cavity effect factor of REN15 is shown to be obviously closer to the results of CE-P than that of FR97

$$
\alpha=\left(1-\varepsilon_{\lim }\right) /\left(1-\varepsilon_{v}\right)=1-\varepsilon_{v} p .
$$
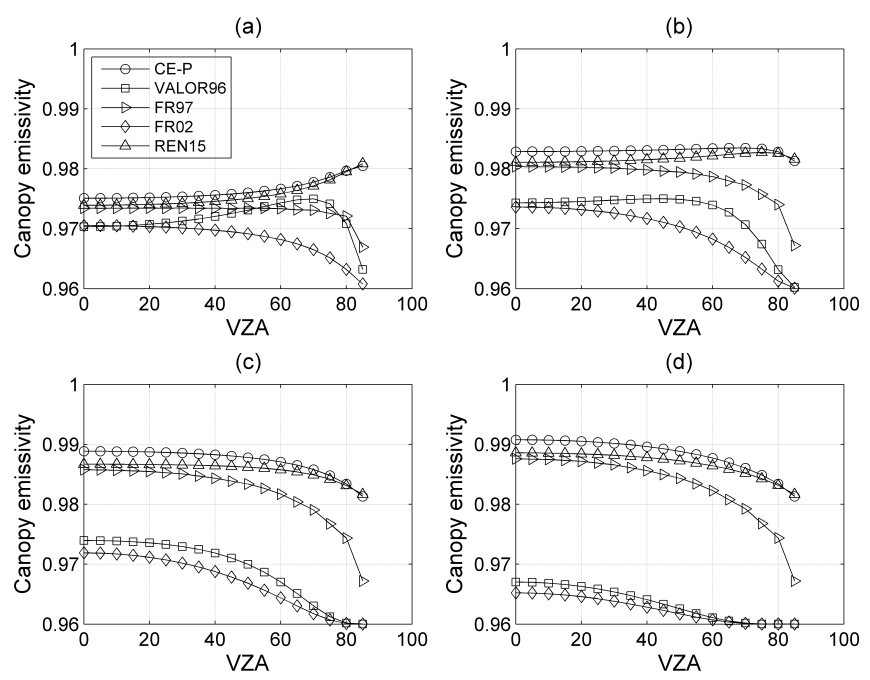

Fig. 31. Canopy emissivity comparison $\left(\varepsilon_{S}=0.96\right.$ and $\left.\varepsilon_{S}=0.96\right)$. (a) $\mathrm{LAI}=0.5$. (b) $\mathrm{LAI}=1$. (c) $\mathrm{LAI}=3$. (d) $\mathrm{LAI}=6$.

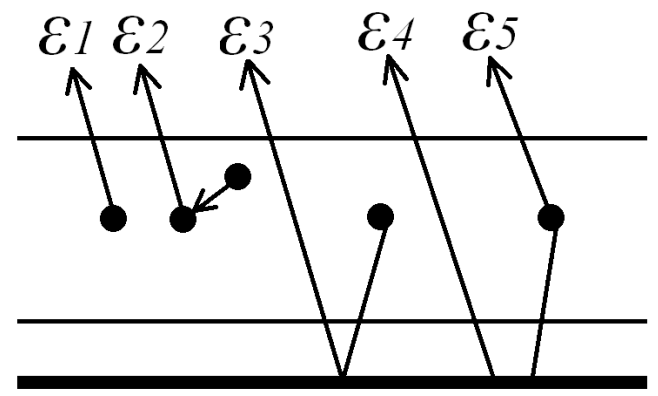

Fig. 32. Illustration of the composition of the canopy emission.

\section{E. Comparisons of VALOR96, FR97, FRO2, and REN15}

The results of CE-P, VALOR96, FR97, FR02, and REN15 are then compared together, as shown in Fig. 30. The overall tendencies of the five models are the same. All four traditional models are underestimated at different levels. The LST estimation algorithm is very sensitive to emissivity errors; generally speaking, a 0.01 error in emissivity will result in an LST error of $1 \mathrm{~K}$ [40]. In addition, an error of 0.01 in broadband emissivity will lead to an error of $3.4 \mathrm{~W} / \mathrm{m}^{2}$ in surface longwave net radiation [41]. VALOR96 and FR02 will underestimate the results by more than 0.01 for large LAI values, making them unacceptable for practice under these circumstances. For small LAIs, after CE-P, REN15 produces the most accurate emissivity over homogeneous canopies. The recommended order for the use of the other models is FR97, FR02, and VALOR96 with the most serious underestimation up to $0.007,0.013$, and 0.014 respectively. The curves of FR02 and VALOR96 always have an intersection point near VZA equal to $50^{\circ}$. FR02 is more accurate before this VZA, and VALOR96 is better after this VZA. It can also be observed from Fig. 30 that the FR97 always drops after the VZA equal to $80^{\circ}$ which leads to a more serious underestimation.

As shown in Fig. 31, an angular variation is still observed when the soil and leaf components have the same emissivities (0.96) because, in this case, the scattering contribution still exists and varies with the viewing angle. 


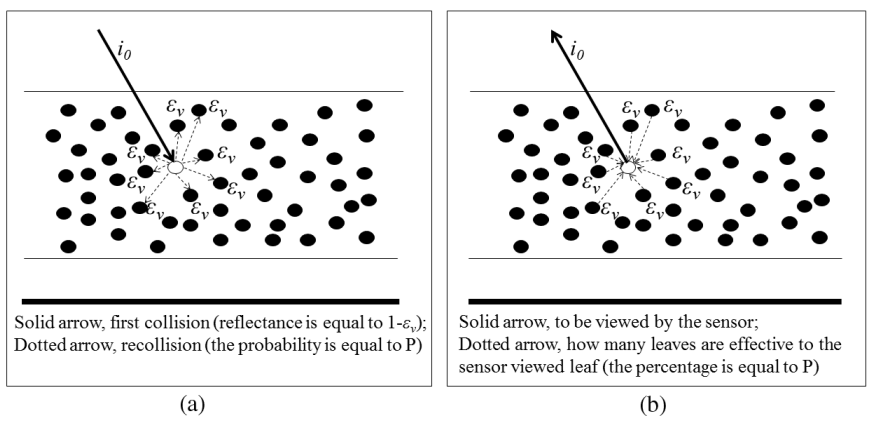

Fig. 33. Illustration of reciprocal theorem. (a) From the perspective of absorption. (b) From the perspective of emission.

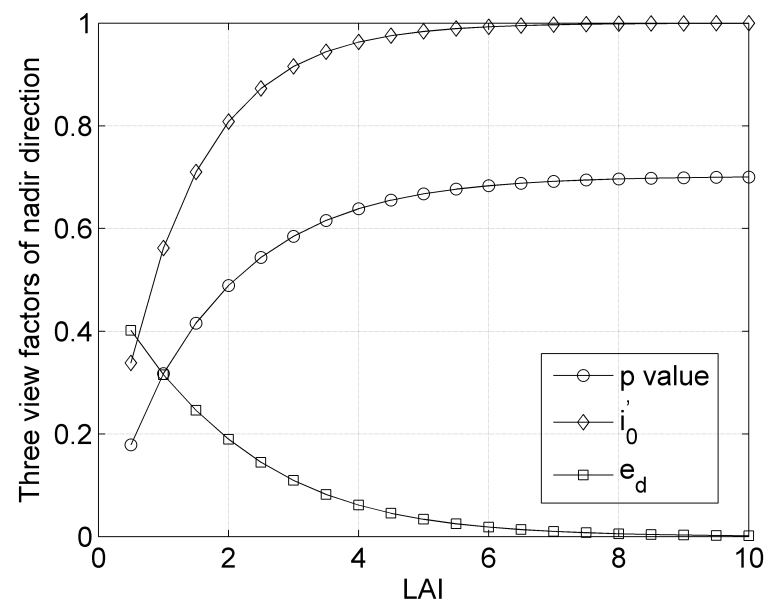

Fig. 34. Comparison of the three view factors with different LAI values.

\section{DisCUSSION AND CONCLUSION}

As the sensitivity analysis result indicated in Section III-B, when the leaf and soil emissivities are equal to 0.98 and 0.94 , respectively, only the direct emissions and the single-scattering effect should be considered from the perspective of active emissions in the TIR band.

There are five terms in total when only considering the direct emissions of the components and the single scatterings between the vegetation and background, as shown in Fig. 32. They are the leaf emission $(\varepsilon 1)$, leaf reflected radiance that comes from the leaf emission $(\varepsilon 2)$, soil reflected radiance that comes from the leaf emission ( $\varepsilon 3)$, soil emission $(\varepsilon 4)$, and leaf reflected radiance that comes from the soil emission $(\varepsilon 5)$.

$\varepsilon 1$ and $\varepsilon 4$ can be calculated directly using the interception fraction [see (3)] and directional gap fraction [see (10)]. $\varepsilon 2, \varepsilon 3$, and $\varepsilon 5$ can be inferred based on three view factors $\left(F_{l l}, F_{l s}\right.$, and $\left.F_{s l}\right)$, as shown in the following equation:

$$
\begin{aligned}
e= & \varepsilon_{1}+\varepsilon_{2}+\varepsilon_{3}+\varepsilon_{4}+\varepsilon_{5} \\
= & \varepsilon_{v} i_{0}+\varepsilon_{v} F_{l l}\left(1-\varepsilon_{v}\right) i_{0}+\varepsilon_{v} F_{l s}\left(1-\varepsilon_{s}\right)\left(1-i_{0}\right) \\
& +\varepsilon_{s}\left(1-i_{0}\right)+\varepsilon_{S} F_{s l}\left(1-\varepsilon_{v}\right) i_{0} .
\end{aligned}
$$

This equation is very similar to the expression of the rowplanted canopy emissivity proposed by Valor and Caselles [38] in 1996. They also proposed three view factors to take into account the fraction of radiation that reaches the ground coming from the vegetation side $(F)$, the fraction of radiation that reaches the vegetation side coming from the ground $(G)$, and the fraction that reaches one side coming from the other side $\left(F^{\prime}\right)$.

Now, we can obtain the equations of the three view factors based on the reciprocal theorem. Fig. 33 explains why $F_{l l}$ is equal to the $p$ value, and we can know that $F_{l s}$ is equal to $i_{0}^{\prime}$ and $F_{s l}$ is equal to $e_{d}$ from the same understanding. Finally, the canopy emissivity of a homogeneous vegetation canopy can be derived as follows:

$$
\begin{aligned}
e=\varepsilon_{v}\left[i_{0}+p\left(1-\varepsilon_{v}\right) i_{0}\right. & \left.+i_{0}^{\prime}\left(1-\varepsilon_{s}\right)\left(1-i_{0}\right)\right] \\
& +\varepsilon_{s}\left[\left(1-i_{0}\right)+e_{d}\left(1-\varepsilon_{v}\right) i_{0}\right] .
\end{aligned}
$$

Equation (25) is the same as (17), which means that we can derive the same result from the perspective of absorption and emission. The equations for $\varepsilon 1, \varepsilon 4$, and $\varepsilon 3$ are widely used in the literature. However, this paper is the first time to use the parameters of $p$ and $e_{d}$ to calculate the contributions of the single scatterings of $\varepsilon 2$ and $\varepsilon 5$, which is one of the most innovative aspects of our work. The two new parameters ( $p$ and $e_{d}$ ) are the function of LAI as $i_{0}^{\prime} . p$ and $i_{0}^{\prime}$ are positively related to LAI, but $e_{d}$ is negatively related to LAI. The trends of $p, i_{0}^{\prime}$, and $e_{d}$ in the nadir direction with different LAIs are shown in Fig. 34.

In this paper, a new directional canopy emissivity estimation method based on spectral invariants is proposed. This paper is the first time to separate the single-scattering effect and multiscattering effect analytically in the TIR band. Furthermore, we analyze the contributions of the multiple-scattering effects with different combinations of component emissivities and find that the total contribution is less than 0.002 when the leaf emissivity is no less than 0.98 and the soil emissivity is no less than 0.94 . The smaller the component emissivities, the larger the overall contributions of multiple scatterings. This contribution is less than 0.005 when the leaf and soil emissivities are equal to 0.90 . We analyzed the spectral library of UCSB and found that $0.94(0.90)$ can be regarded as the boundary value of leaf (soil) emissivity. Therefore, the simplified model is practical in the applications. Within this boundary range, three main conclusions can be deduced from the analysis and validation.

1) The multiple scatterings between the vegetation and the background can be ignored within a homogeneous canopy when the leaf (soil) emissivity is no less than $0.94(0.90)$.

2) The second-order and beyond scatterings within the vegetation can be ignored within a homogeneous canopy when the leaf (soil) emissivity is no less than $0.94(0.90)$.

3) The single-scattering effect within a homogeneous canopy should be considered and can be obtained using the three view factors proposed in CE-P. The hemispherical average intercept probability $\left(i_{0}^{\prime}\right)$ can be used to calculate how many leaves are effective to background. The fraction of radiation that reaches the leaf coming from other leaves can be calculated using the parameter of the $p$ value, and the fraction of radiation that reaches the leaf coming from the background can be calculated 
using the escape probability $\left(e_{d}\right)$. The last two components are the most innovative points of this paper, and it is a significant progress to represent $p$ value to replace the concept of the cavity effect factor, which has been widely used for 20 years as an empirical solution.

Only the spherical LAD is considered in this paper for the simplification of the calculation of the $p$ value. More LADs will be considered in the future. Some crops and grassland are homogeneous and are suitable for our new canopy emissivity model. However, most forests in the nature are heterogeneous. Further investigation is required to extend our model to heterogeneous canopies. Multiscale $p$ value may be adopted to achieve the extension from homogeneous canopy to heterogeneous canopy, and DART will be the best choice for us to do cross validation because of its good performance in the simulation of 3-D canopies. After the separation of the contributions of leaves and background, as we suggested, land surface component temperatures can potentially be more accurately estimated based on the new model, which will be the focus of future works.

\section{REFERENCES}

[1] M. C. Anderson, J. M. Norman, W. P. Kustas, R. Houborg, P. J. Starks, and N. Agam, "A thermal-based remote sensing technique for routine mapping of land-surface carbon, water and energy fluxes from field to regional scales," Remote Sens. Environ., vol. 112, no. 12, pp. 4227-4241, Dec. 2008.

[2] L. Jia, Z.-L. Li, M. Menenti, Z. Su, W. Verhoef, and Z. Wan, "A practical algorithm to infer soil and foliage component temperatures from bi-angular ATSR-2 data," Int. J. Remote Sens., vol. 24, no. 23, pp. 4739-4760, 2003.

[3] Z.-L. Li et al., "Satellite-derived land surface temperature: Current status and perspectives," Remote Sens. Environ., vol. 131, pp. 14-37, Apr. 2013.

[4] Y. Du, Q. Liu, L. Chen, Q. Liu, and T. Yu, "Modeling directional brightness temperature of the winter wheat canopy at the ear stage," IEEE Trans. Geosci. Remote Sens., vol. 45, no. 11, pp. 3721-3739, Nov. 2007.

[5] Q. Liu, H. Huang, W. Qin, K. Fu, and X. Li, "An extended 3-D radiosity-graphics combined model for studying thermal-emission directionality of crop canopy," IEEE Trans. Geosci. Remote Sens., vol. 45, no. 9, pp. 2900-2918, Sep. 2007.

[6] H. Huang, Q. Liu, W. Qin, Y. Du, and X. Li, "Temporal patterns of thermal emission directionality of crop canopies," J. Geophys. Res. Atmos., vol. 116, no. D06114, pp. 1-18, Mar. 2011.

[7] H. Ren, G. Yan, R. Liu, F. Nerry, Z.-L. Li, and R. Hu, "Impact of sensor footprint on measurement of directional brightness temperature of row crop canopies," Remote Sens. Environ., vol. 134, pp. 135-151, Jul. 2013.

[8] D. S. Kimes, "Remote sensing of row crop structure and component temperatures using directional radiometric temperatures and inversion techniques," Remote Sens. Environ., vol. 13, no. 1, pp. 33-55, Mar. 1983.

[9] J. A. Sobrino and J. Cuenca, "Angular variation of thermal infrared emissivity for some natural surfaces from experimental measurements," Appl. Opt., vol. 38, no. 18, pp. 3931-3936, Jun. 1999.

[10] V. García-Santos, E. Valor, V. Caselles, M. Á. Burgos, and C. Coll, "On the angular variation of thermal infrared emissivity of inorganic soils," J. Geophys. Res.-Atmos., vol. 117, no. D19116, pp. 1-18, Oct. 2012.

[11] G. Fontanilles, X. Briottet, S. Fabre, and T. Trémas, "Thermal infrared radiance simulation with aggregation modeling (TITAN): An infrared radiative transfer model for heterogeneous three-dimensional surface-Application over urban areas," Appl. Opt., vol. 47, no. 31, pp. 5799-5810, Nov. 2008.

[12] J.-P. Lagouarde et al., "Modelling daytime thermal infrared directional anisotropy over Toulouse city centre," Remote Sens. Environ., vol. 114, no. 1 , pp. $87-105$, Jan. 2010
[13] J. Cheng, S. Liang, F. Weng, J. Wang, and X. Li, "Comparison of radiative transfer models for simulating snow surface thermal infrared emissivity," IEEE J. Sel. Topics Appl. Earth Observ. Remote Sens., vol. 3, no. 3, pp. 323-336, Sep. 2010.

[14] Y. Shi, "Thermal infrared inverse model for component temperatures of mixed pixels," Int. J. Remote Sens., vol. 32, no. 8, pp. 2297-2309, 2011.

[15] B. Cao, Q. Liu, Y. Du, H. Li, H. Wang, and Q. Xiao, "Modeling directional brightness temperature over mixed scenes of continuous crop and road: A case study of the Heihe River Basin," IEEE Geosci. Remote Sens. Lett., vol. 12, no. 2, pp. 234-238, Feb. 2015.

[16] J. A. Sobrino, J. Jiménez-Muñoz, and W. Verhoef, "Canopy directional emissivity: Comparison between models," Remote Sens. Environ., vol. 99, no. 3, pp. 304-314, Nov. 2005.

[17] W. C. Snyder and Z. Wan, "BRDF models to predict spectral reflectance and emissivity in the thermal infrared," IEEE Trans. Geosci. Remote Sens., vol. 36, no. 1, pp. 214-225, Jan. 1998.

[18] C. Francois, C. Ottlé, and L. Prévot, "Analytical parameterization of canopy directional emissivity and directional radiance in the thermal infrared. Application on the retrieval of soil and foliage temperatures using two directional measurements," Int. J. Remote Sens., vol. 18, no. 12, pp. 2587-2621, Aug. 1997.

[19] C. François, "The potential of directional radiometric temperatures for monitoring soil and leaf temperature and soil moisture status," Remote Sens. Environ., vol. 80, no. 1, pp. 122-133, Apr. 2002.

[20] W. Verhoef, L. Jia, Q. Xiao, and Z. Su, "Unified optical-thermal fourstream radiative transfer theory for homogeneous vegetation canopies," IEEE Trans. Geosci. Remote Sens., vol. 45, no. 6, pp. 1808-1822, Jun. 2007.

[21] H. Z. Ren et al., "Performance evaluation of four directional emissivity analytical models with thermal SAIL model and airborne images," Opt. Express, vol. 23, no. 7, pp. A346-A360, Apr. 2015.

[22] L. Prévot, "Modélisation des échanges radiatifs au sein des couverts végétaux. Application a la télédétection. Validation sur un couvert de mais," Ph.D. dissertation, Univ. Paris VI, Paris, France, 1985.

[23] P. Guillevic, J. P. Gastellu-Etchegorry, J. Demarty, and L. Prévot, "Thermal infrared radiative transfer within three-dimensional vegetation covers," J. Geophys. Res.-Atmos., vol. 108, no. D8, pp. 42-48, 2003.

[24] J.-P. Gastellu-Etchegorry et al., "DART: Recent advances in remote sensing data modeling with atmosphere, polarization, and chlorophyll fluorescence," IEEE J. Sel. Topics Appl. Earth Observ. Remote Sens., vol. 10, no. 6, pp. 2640-2649, Jun. 2017.

[25] Y. Knyazikhin, J. V. Martonchik, R. B. Myneni, D. J. Diner, and S. W. Running, "Synergistic algorithm for estimating vegetation canopy leaf area index and fraction of absorbed photosynthetically active radiation from MODIS and MISR data," J. Geophys. Res., Atmos., vol. 103, no. D24, pp. 32257-32275, 1998.

[26] J. W. Salisbury, A. Wald, and D. M. Daria, "Thermal-infrared remote sensing and Kirchhoff's law: 1. Laboratory measurements," J. Geophys. Res.-Solid Earth, vol. 99, no. B6, pp. 11897-11911, Jun. 1994.

[27] M. Rautiainen and P. Stenberg, "Application of photon recollision probability in coniferous canopy reflectance simulations," Remote Sens. Environ., vol. 96, no. 1, pp. 98-107, May 2005.

[28] M. Mõttus, "Photon recollision probability in discrete crown canopies," Remote Sens. Environ., vol. 110, no. 2, pp. 176-185, Sep. 2007.

[29] W. Fan, Y. Liu, X. Xu, G. Chen, and B. Zhang, "A new FAPAR analytical model based on the law of energy conservation: A case study in China," IEEE J. Sel. Topics Appl. Earth Observ. Remote Sens., vol. 7, no. 9, pp. 3945-3955, Sep. 2014.

[30] P. Stenberg, "Simple analytical formula for calculating average photon recollision probability in vegetation canopies," Remote Sens. Environ., vol. 109, no. 2, pp. 221-224, Jul. 2007.

[31] P. Stenberg, P. Lukeš, M. Rautiainen, and T. Manninen, "A new approach for simulating forest albedo based on spectral invariants," Remote Sens. Environ., vol. 137, pp. 12-16, Oct. 2013.

[32] X. Xu, W. Fan, J. Li, P. Zhao, and G. Chen, "A unified model of bidirectional reflectance distribution function for the vegetation canopy," Sci. China-Earth Sci., vol. 60, no. 3, pp. 463-477, Mar. 2017.

[33] P. Stenberg, M. Mõttus, and M. Rautiainen, "Photon recollision probability in modelling the radiation regime of canopies-A review," Remote Sens. Environ., vol. 183, pp. 98-108, Sep. 2016.

[34] H. Li et al., "Evaluation of the VIIRS and MODIS LST products in an arid area of Northwest China," Remote Sens. Environ., vol. 142, pp. 111-121, Feb. 2014.

[35] T. Nilson, "A theoretical analysis of the frequency of gaps in plant stands," Agricult. Meteorol., vol. 8, pp. 25-38, 1971. 
[36] W. Verhoef, "Light scattering by leaf layers with application to canopy reflectance modeling: The SAIL model," Remote Sens. Environ., vol. 16, no. 2, pp. $125-141$, Oct. 1984.

[37] J. M. Norman and F. Becker, "Terminology in thermal infrared remote sensing of natural surfaces," Agricult. Forest Meteorol., vol. 77, nos. 3-4, pp. 153-166, Dec. 1995.

[38] E. Valor and V. Caselles, "Mapping land surface emissivity from NDVI: Application to European, African, and South American areas," Remote Sens. Environ., vol. 57, no. 3, pp. 167-184, 1996.

[39] Z.-L. Li et al., "Land surface emissivity retrieval from satellite data," Int. J. Remote Sens., vol. 34, nos. 9-10, pp. 3084-3127, May 2013.

[40] J. M. Galve, C. Coll, V. Caselles, and E. Valor, "An atmospheric radiosounding database for generating land surface temperature algorithms," IEEE Trans. Geosci. Remote Sens., vol. 46, no. 5, pp. 1547-1557, May 2008.

[41] J. Cheng and S. Liang, "Effects of thermal-infrared emissivity directionality on surface broadband emissivity and longwave net radiation estimation," IEEE Geosci. Remote Sens. Lett., vol. 11, no. 2, pp. 499-503, Feb. 2014.

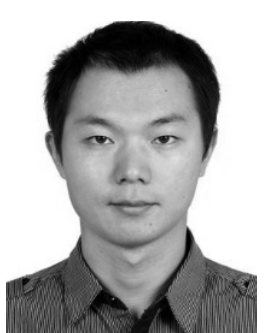

Biao Cao received the B.Sc. degree in geographic information system from Beijing Forestry University, Beijing, China, in 2009, and the Ph.D. degree in cartography and geographic information system from the Institute of Remote Sensing and Digital Earth, Chinese Academy of Sciences (CAS), Beijing, in 2014.

Since Dec. 2017, he has been a Visiting Scientist with the DART Group of CESBIO (CNRS, UPS, CNES, IRD, and INRA), Toulouse, France. He is currently a Research Assistant with the State Key Laboratory of Remote Sensing Science, Institute of Remote Sensing and Digital Earth, CAS. His research interests include the thermal radiation transfer modeling for complex land surface and longwave upward radiation estimation with considering the emission directionality.

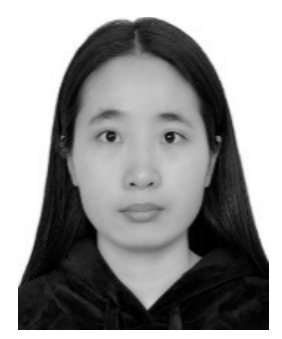

Mingzhu Guo received the B.Sc. degree in remote sensing of science and technology from the Shandong University of Science and Technology, Qingdao, China, in 2016. She is currently pursuing the master's degree in photogrammetry and remote sensing with Peking University, Beijing, China, with a focus on thermal infrared remote sensing and vegetation remote sensing.

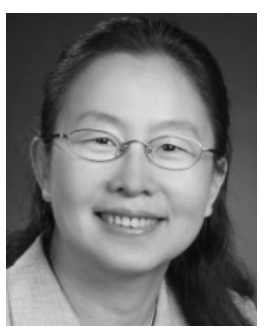

Wenjie Fan received the Ph.D. degree in physical geography from the College of Urban and Environmental Sciences, Peking University (PKU), Beijing, China, in 2000.

She completed the post-doctorate research at the Institute of Remote Sensing and Geographic Information System, PKU, in 2002, where she has been an Associate Professor since 2005. She has also been the PI of more than six national natural funds and other research projects. She has continued to involve and manage research projects on vegetation remote sensing, scale effects of remote sensing, hyperspectral remote sensing, and application of remote sensing in ecology and environment management for 18 years. She has authored over 80 papers (over 30 papers indexed by SCI).

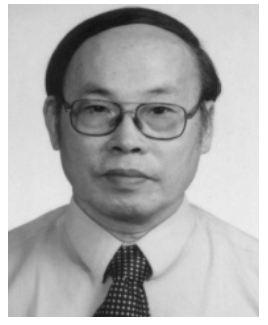

Xiru Xu received the B.Sc. degree from the Department of Geophysics, Peking University (PKU), Beijing, China, in 1960.

From 1981 to 1983, he was a Visiting Scholar with the Electrical Engineering and Computer Science Department, MIT, Cambridge, MA, USA. He is currently a Professor with the Institute of Remote Sensing and Geographic Information System, PKU. $\mathrm{He}$ has continued to involve and manage research projects on quantitative remote sensing, microwave remote sensing, and vegetation remote sensing for more than 30 years. He is one of the first generation of remote sensing science researchers in China.

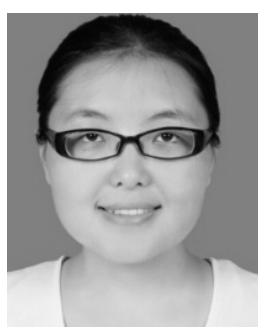

Jingjing Peng received the Ph.D. degree in cartography and geographic information system from the Institute of Remote Sensing and Digital Earth, Chinese Academy of Sciences, Beijing, China, in 2014.

She completed the post-doctorate research at the Institute of Remote Sensing and Geographic Information System, Peking University, in 2016. She is currently a Post-Doctoral Researcher with the Earth System Science Interdisciplinary Center, University of Maryland, College Park, MD, USA. She is involved in JPSS/GOES-R operational albedo products and physical albedo model development, ice-albedo effect, and vegetation remote sensing.

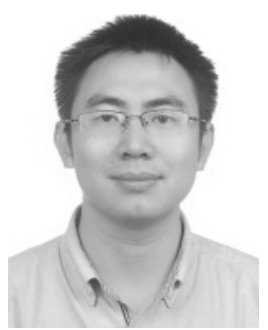

Huazhong Ren received the Ph.D. degree from Beijing Normal University, Beijing, China, and from Université de Strasbourg, Strasbourg, France, in 2013

Since 2016, he has been an Assistant Professor with the Institute of Remote Sensing and Geographic Information System, School of Earth and Space Sciences, Peking University, Beijing. His research interests include land surface temperature/emissivity estimate from thermal infrared remote sensing data.

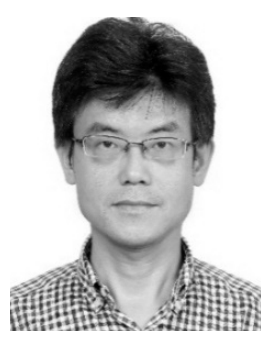

Yongming Du received the B.Sc. degree in geography from Yantai Normal College, Yantai, China, in 1999, the M.Sc. degree in cartography and remote sensing from Peking University, Beijing, China, in 2003, and the Ph.D. degree in cartography and remote sensing from the Institute of Remote Sensing Applications, Chinese Academy of Sciences (CAS), Beijing, in 2006.

$\mathrm{He}$ is currently an Associate Professor with the Institute of Remote Sensing and Digital Earth, CAS. His research interests include the radiation transfer model of vegetation.

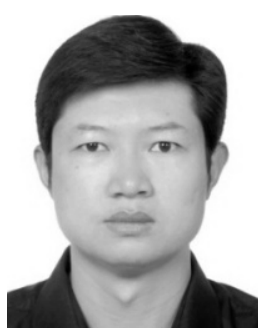

Hua $\mathbf{L i}$ received the B.Sc. degree in geographic information system from the Xi'an University of Science and Technology, Xi'an, China, in 2004, the M.Sc. degree in cartography and geographic information system from Central South University, Changsha, China, in 2007, and the Ph.D. degree in cartography and geographic information system from the Institute of Remote Sensing Applications, Chinese Academy of Sciences (CAS), Beijing, China, in 2010.

$\mathrm{He}$ is currently an Associate Researcher with the State Key Laboratory of Remote Sensing Science, Institute of Remote Sensing and Digital Earth, CAS. His research interests include the retrieval and validation of land surface temperature/emissivity from satellite data. 


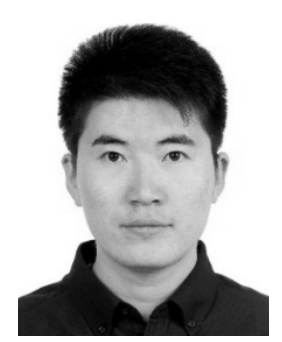

Zunjian Bian received the B.Sc. degree in geographic information system from the China University of Petroleum, Qingdao, China, in 2013. He is currently pursuing the Ph.D. degree with the University of Chinese Academy of Sciences, Beijing, China.

His research interests include the retrieval of component temperatures and 3-D radiative transfer modeling in thermal infrared domain.

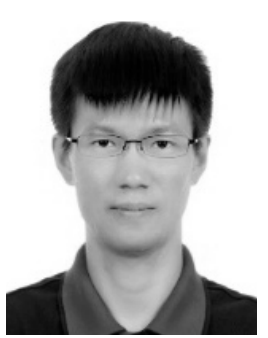

Tian Hu received the B.E. degree in remote sensing science and technology from Wuhan University, Wuhan, China, in 2013. He is currently pursuing the Ph.D. degree with the University of Chinese Academy of Sciences, Beijing, China, and with the Environment Futures Research Institute, School of Natural Sciences, Griffith University, Nathan, QLD, Australia.

His research interests include the retrieval and validation of land surface temperature/emissivity, and the estimation of the land surface upwelling longwave radiation using remote sensing methods and combining deep learning with remote sensing.

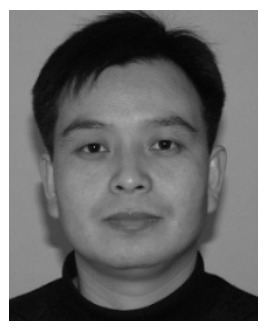

Qing Xiao received the bachelor's degree from Jilin University, Changchun, China, in 1993, and the M.S. and Ph.D. degrees in remote sensing from the Institute of Remote Sensing Applications, Chinese Academy of Sciences (CAS), Beijing, China, in 1996 and 2002, respectively.

$\mathrm{He}$ is currently a Professor with the State Key Laboratory of Remote Sensing Science, Institute of Remote Sensing and Digital Earth, CAS. His research interests include the quantitative processing and application of thermal infrared data and the validation of remote sensing products.

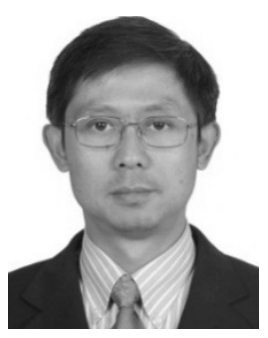

Qinhuo Liu received the B.Sc. degree in hydrogeology and engineering geology from Southwest Jiaotong University, Chengdu, China, in 1988, and the M.Sc. degree in cartography and remote sensing and the Ph.D. degree in atmospheric physics from Peking University, Beijing, China, in 1994 and 1997, respectively.

He was a Short-Term Visiting Scholar with INRA, Paris, France, in 1998, Boston University, Boston, MA, USA, in 1999, the University of Maryland, College Park, MD, USA, in 2004, and George Mason University, Fairfax, VA, USA, in 2010. Since 1997, he has been with the Institute of Remote Sensing and Digital Earth, Chinese Academy of Sciences, Beijing, where he is currently a Professor and the Deputy Director with the State Key Laboratory of Remote Sensing Science. His research interests include the radiation transfer modeling for optical remote sensing and terrestrial parameter inversion from multisource remote sensing data.

Dr. Liu is a member of the American Geophysical Union and the IEEE Geoscience and Remote Sensing Society. 